\title{
Plasmoid and Kelvin-Helmholtz instabilities in Sweet-Parker current sheets
}

\author{
N. F. Loureiro, ${ }^{1}$ A. A. Schekochihin, ${ }^{2}$ and D. A. Uzdensky ${ }^{3}$ \\ ${ }^{1}$ Associação EURATOM/IST, Instituto de Plasmas e Fusão Nuclear - Laboratório Associado, \\ Instituto Superior Técnico, Universidade Técnica de Lisboa, 1049-001 Lisboa, Portugal \\ ${ }^{2}$ Rudolf Peierls Centre for Theoretical Physics, University of Oxford, Oxford OX1 3NP, UK \\ ${ }^{3}$ Center for Integrated Plasma Studies, Physics Department, \\ University of Colorado, Boulder CO 80309, USA
}

(Dated: May 24, 2018)

\begin{abstract}
A 2D linear theory of the instability of Sweet-Parker (SP) current sheets is developed in the framework of Reduced MHD. A local analysis is performed taking into account the dependence of a generic equilibrium profile on the outflow coordinate. The plasmoid instability [Loureiro et al., Phys. Plasmas 14, 100703 (2007)] is recovered, i.e., current sheets are unstable to the formation of a large-wave-number chain of plasmoids $\left(k_{\max } L_{\mathrm{CS}} \sim S^{3 / 8}\right.$, where $k_{\max }$ is the wave-number of fastest growing mode, $S=L_{\mathrm{CS}} V_{A} / \eta$ is the Lundquist number, $L_{\mathrm{CS}}$ is the length of the sheet, $V_{A}$ is the Alfvén speed and $\eta$ is the plasma resistivity), which grows super-Alfvénically fast $\left(\gamma_{\max } \tau_{A} \sim S^{1 / 4}\right.$, where $\gamma_{\max }$ is the maximum growth rate, and $\left.\tau_{A}=L_{\mathrm{CS}} / V_{A}\right)$. For typical background profiles, the growth rate and the wave-number are found to increase in the outflow direction. This is due to the presence of another mode, the Kelvin-Helmholtz $(\mathrm{KH})$ instability, which is triggered at the periphery of the layer, where the outflow velocity exceeds the Alfvén speed associated with the upstream magnetic field. The KH instability grows even faster than the plasmoid instability, $\gamma_{\max } \tau_{A} \sim k_{\max } L_{\mathrm{CS}} \sim S^{1 / 2}$. The effect of viscosity $(\nu)$ on the plasmoid instability is also addressed. In the limit of large magnetic Prandtl numbers, $P m=\nu / \eta$, it is found that $\gamma_{\max } \sim S^{1 / 4} \mathrm{Pm}^{-5 / 8}$ and $k_{\max } L_{\mathrm{CS}} \sim S^{3 / 8} \mathrm{Pm}^{-3 / 16}$, leading to the prediction that the critical Lundquist number for plasmoid instability in the $\mathrm{Pm} \gg 1$ regime is $S_{\text {crit }} \sim 10^{4} \mathrm{Pm}^{1 / 2}$. These results are verified via direct numerical simulation of the linearized equations, using a new, analytical 2D SP equilibrium solution.
\end{abstract}

PACS numbers: 52.35.Vd, 52.35.Py, 94.30.cp, 96.60.Iv

\section{INTRODUCTION}

Magnetic reconnection [1-4] is a ubiquitous plasma physics phenomenon, characterized by the rapid reconfiguration of the magnetic-field topology. Solar flares [5] and magnetospheric substorms [6] are two prominent examples of events where reconnection plays a key role. Plasma dynamics in many laboratory experiments is also critically determined by magnetic reconnection; examples are the sawtooth [7] and the tearing instabilities [8, 9] in magnetic-confinement-fusion devices, or the reconnection of high-energy-density, laser-produced plasma bubbles 10 12.

Along with fast reconnection rates, many observations [13] of magnetic reconnection phenomena display one intriguing feature: the formation, and subsequent ejection from the current sheet, of coherent secondary structures, often referred to as plasmoids (also known as blobs, flux ropes or secondary magnetic islands). There is abundant direct evidence for the presence of these structures in the Earth's magnetotail [14 16] and in solar flares [17 22]. In magnetic confinement fusion devices, plasmoid generation seems to be less certain, though there are reports of the observation of secondary magnetic structures correlated to $m / n=1 / 1$ and $m / n=2 / 1$ magnetic islands on the TEXTOR [23, 24] and JET [25] tokamaks. On TEXTOR, high-resolution measurements of electron temperature fluctuations show structures which hint at plasmoid formation during saw- tooth crashes 26 28]. Finally, recent laser-plasma experiments where reconnection is conjectured to occur also show evidence for plasmoid formation 11, 12].

Direct numerical simulations of reconnection processes concur with observations in displaying ubiquitous evidence for plasmoid formation. Plasmoids have been reported in numerical simulations using various physical models, ranging from kinetic 29 33 to Hall-MHD 34, 35] and to single fluid MHD [36 49]. Plasmoid formation has also been reported in numerical simulations of reconnection in relativistic plasmas, both resistive [50] and kinetic 51 54]. Numerical studies tailored to address specific reconnection contexts such as the solar corona 55 58], the Earth's magnetotail [59, 60], magnetic young stellar objects [61 63], fusion experiments [36, 39] and laser-plasma interactions [64], though different from each other in a number of details, again all appear to agree on the basic fact that plasmoids are generated in reconnecting current sheets.

The plasmoid dynamics inferred from observations and seen in numerical simulations strongly suggest the very opposite of the laminar, steady-state reconnection scenarios that have dominated the field for much of its history [the Sweet-Parker (SP) [65, 66] and the Petschek 67] models, and, more recently, the Hall reconnection paradigm [68]]. Magnetic reconnection in the presence of plasmoids appears to be a highly timedependent, bursty process, which can only be described in a statistical manner [49, 58, 69-72]. Furthermore, in 
addition to their key role in setting the reconnection rate in both laminar 32, 33, 35, 42, 45 47, 49, 69, 71 and turbulent [44, 48] plasmas, there is numerical and observational evidence that plasmoids may be critical in explaining electron acceleration in reconnection sites [16, 73 75].

In a previous paper 76] (henceforth referred to as $\mathrm{Pa}$ per I) we attempted to understand the origin of plasmoid formation in reconnection sites by analysing the linear stability of large-aspect-ratio, SP current sheets. These were found to be violently unstable to the formation of plasmoid chains, the fastest growing wave number scaling as $k_{\max } L_{\mathrm{CS}} \sim S^{3 / 8}$, with corresponding growth rate $\gamma_{\max } \tau_{A} \sim S^{1 / 4}$, where $L_{\mathrm{CS}}$ is the length of the current layer, $\tau_{A}=L_{\mathrm{CS}} / V_{A}$ is the Alfvén time ( $V_{A}$ is the Alfvén speed) and $S$ is the Lundquist number, $S=L_{\mathrm{CS}} V_{A} / \eta$, where $\eta$ is the magnetic diffusivity. Since $S \gg 1$ in most applications of interest, this theory predicts the formation of multidinous plasmoids growing super-Alfvénically; the immediate implication is that stable reconnecting current sheets at large values of the Lundquist number cannot exist. These results have since been confirmed in direct numerical simulations [43, 77], and extended to account for the effect of a finite component of the magnetic field perpendicular to the reconnection plane [78] and into the two-fluid regime [79].

The analysis of Paper I considered a very simplified model background equilibrium, intended to retain only what we viewed as the most important features of a SP current sheet: a reconnecting magnetic field, $\boldsymbol{B}_{e q}=\left(0, B_{y}(x)\right)$ ( $x$ is the inflow direction, $y$ the outflow direction) and an incompressible flow defined by the stream function $\phi_{e q}=\Gamma_{0} x y$, where $\Gamma_{0}=V_{A} / L_{\mathrm{CS}}$ is the flow shearing rate. The analytical derivation in Paper I did not, therefore, take into account potentially important effects, such as the variation of the reconnecting magnetic field and of the outflow speed along the layer (i.e., along the $y$-direction in our chosen geometry), or the reconnected magnetic field.

In this work, we generalize the results of Paper I to a more realistic, two-dimensional model of the current sheet. Using an approach in the spirit of WKB theory (justified by the expectation that the most unstable wave-number will be very large, $k_{\max } L_{\mathrm{CS}} \gg 1$ ), we derive the dispersion relation for the plasmoid instability as a slow function of the position along the sheet, $y_{0}$. We find that the scalings of the maximum growth rate $\left(\gamma_{\max }\right)$ and wave-number $\left(k_{\max }\right)$ derived in Paper I hold true in a central, finite-sized patch of the current sheet; however, the growth rate and wave number are now parametrized nontrivially by $y_{0}$. Surprisingly, we also discover that for a generic background equilibrium configuration, the maximum growth rate and wave-number of the instability increase with $y_{0}$ (i.e., outwards). As we show in this paper, a special point exists, $y_{0, c r i t}$, beyond which the assumptions invoked in our calculation break down. This is the Alfvén Mach point of the system, where the magnitude of the outflow velocity (an increasing function of $y_{0}$ ) becomes equal to the value of the Alfvén speed based on the upstream magnetic field (a decreasing function of $y_{0}$ ). Beyond that point, the current sheet becomes unstable to a different mode: the Kelvin-Helmholtz (KH) instability, whose growth rate and wave-number dependence we also derive analytically.

The other main result of this paper is the study of the effect of a large viscosity $\nu$ (parametrized by the magnetic Prandtl number $P m=\nu / \eta \gg 1)$ on the plasmoid instability. The large-Prandtl-number regime is pertinent to various astrophysical applications, e.g., the interstellar medium [80], and to fusion plasmas [36], and so it is important to understand how large $\mathrm{Pm}$ affects plasmoid formation and dynamics.

Our analytical results are complemented with a direct numerical solution of the full set of linearized equations.

This paper is organized as follows. In section [II, we present a heuristic derivation of our main results. A more rigorous approach to the problem begins in section [II], where the equations to be solved are laid out and the expected properties of the background equilibrium are discussed (a more quantitative discussion of the constraints that the background equilibrium should satisfy can be found in Appendix A, where an analytical 2D $\mathrm{SP}$-like current-sheet equilibrium is obtained). The core of the analytical calculation is presented in section IV. The KH instability of the current sheet is derived in section V. Results of the direct numerical solution of the linear equations are presented in section VI The effect of viscosity on the instability is addressed in sectionVIB. Finally, a discussion of the results and conclusions can be found in section VII

\section{HEURISTIC DERIVATION}

In this section, we show how the main results of this paper can be derived in a simple (albeit non-rigorous) way. A reader uninterested in the formal mathematical details can skip to section VI after this section.

\section{A. Plasmoid instability}

The fastest growth rate of the plasmoid instability can be obtained from the usual tearing mode formulae as follows [45].

In the small $\Delta^{\prime}$ limit, where $\Delta^{\prime}$ is the usual tearing mode instability parameter, the standard (FKR) tearing mode dispersion relation is 8 ]

$$
\gamma \sim \tau_{H}^{-2 / 5} \tau_{\eta}^{-3 / 5}\left(\Delta^{\prime} a\right)^{4 / 5}
$$

where $a$ is the characteristic equilibrium magnetic field length scale, $\tau_{\eta}=a^{2} / \eta$ is the resistive diffusion time and $\tau_{H}=1 / k B_{0}$ is the hydrodynamic time. In the opposite limit of large $\Delta^{\prime}$ [81],

$$
\gamma \sim \tau_{H}^{-2 / 3} \tau_{\eta}^{-1 / 3}
$$


In both cases, the width of the resistive (or inner) boundary layer is

$$
\delta_{\text {inner }} / a \sim\left(\gamma \tau_{H}^{2} \tau_{\eta}^{-1}\right)^{1 / 4}
$$

To find the fastest growing mode, let us assume a simple Harris-sheet equilibrium, $B_{y}=B_{0} \tanh (x / a)$; then, for $k a \ll 1$, as we expect to be the case for $k=k_{\max }$, we have $\Delta^{\prime} a \sim 1 / k a$. Substituting this expression in Eq. (1), we find that it yields $\gamma \propto k^{-2 / 5}$, whereas from Eq. (2) we have $\gamma \sim k^{2 / 3}$. Approximate expressions for the largest growth rate and corresponding wave number can therefore be found by balancing Eqs. (1) and (2). This gives

$$
\begin{aligned}
k_{\max } a & \sim\left(a / B_{0}\right)^{1 / 4} \tau_{\eta}^{-1 / 4}, \\
\gamma_{\max } & \sim\left(a / B_{0}\right)^{-1 / 2} \tau_{\eta}^{-1 / 2} .
\end{aligned}
$$

The corresponding inner-layer width is

$$
\delta_{\text {inner }} / a \sim\left(a B_{0} / \eta\right)^{-1 / 4} .
$$

In order to apply these scalings to a SP current sheet, we rescale the equilibrium length scale $a$ to the sheet thickness:

$$
a \equiv \delta_{\mathrm{CS}} \sim L_{\mathrm{CS}} S^{-1 / 2}
$$

Noting that the plasma outflow speed in a SP current sheet is $V_{A}=B_{0}$, we obtain

$$
\begin{aligned}
k_{\max } L_{\mathrm{CS}} & \sim S^{3 / 8}, \\
\gamma_{\max } \tau_{A} & \sim S^{1 / 4}, \\
\delta_{\text {inner }} / \delta_{\mathrm{CS}} & \sim S^{-1 / 8},
\end{aligned}
$$

where $\tau_{A}=L_{\mathrm{CS}} / V_{A}$. These predictions are in agreement with the results of Paper I 76].

We can now use these results to estimate the critical value of the Lundquist number, $S_{\text {crit }}$, below which we expect SP current sheets to be stable. The underlying reasoning is that for the plasmoid instability to be triggered we must have $\gamma_{\max } \tau_{A} \gg 1, k_{\max } L_{\mathrm{CS}} \gg 1$ and $\delta_{\text {inner }} / \delta_{\mathrm{CS}} \ll 1$, i.e., the instability has to grow faster than the characteristic outflow time and it has to fit inside the current sheet, both along (thus the restriction on $k_{\max }$ ) and across (thus the restriction on $\delta_{\text {inner }}$ ). Of all these conditions, the most stringent is that on the width of the inner layer, since it bears the weakest $S$ dependence. Therefore, if we require (non-rigorously!) that $\delta_{\text {inner }} / \delta_{\mathrm{CS}} \sim 1 / 3$ at the very most, then Eq. (10) would yield $S_{\text {crit }} \sim 10^{4}$. This is consistent with numerical simulations 38, 39, 41, 43.

\section{B. Plasmoid instability at large Pm}

One limitation of Paper I was that plasma viscosity was neglected. At low values of the magnetic Prandtl number, $P m=\nu / \eta$ [relevant to the interiors of stars and planets, or liquid metal laboratory dynamos, for example [see [82] and references therein)], the presence of viscosity should not change our results substantially. In contrast, for $P m \gg 1$, as is often found in fusion plasmas [36], warm interstellar and intracluster media [80, 83], etc., both the SP scalings and the tearing and kink modes scalings change [84], and so, therefore, will the plasmoid instability.

Let us work out the plasmoid scalings in the large$\mathrm{Pm}$ limit in a similar way to that just presented for the invisicid case. Instead of the FKR [8] and Coppi et al. 81] results, we now use the corresponding formulae valid for $P m \gg 1$, i.e., the the so-called "visco-tearing" (low $\Delta^{\prime}$ ) and "visco-resistive kink" (large $\Delta^{\prime}$ ) derived by Porcelli [84].

At low $\Delta^{\prime}$ (the visco-tearing mode), we have

$$
\gamma \sim \tau_{H}^{-1 / 3} \tau_{\eta}^{-5 / 6} \tau_{\nu}^{1 / 6} \Delta^{\prime} a
$$

where $\tau_{\nu}=a^{2} / \nu$ is the viscous diffusion time. At $\Delta^{\prime} \rightarrow$ $\infty$ (the visco-resistive kink), the growth rate is

$$
\gamma \sim \tau_{H}^{-2 / 3} \tau_{\eta}^{-2 / 3} \tau_{\nu}^{1 / 3}
$$

The corresponding inner-layer width is

$$
\delta_{\text {inner }} / a \sim\left[\tau_{H}^{2} /\left(\tau_{\eta} \tau_{\nu}\right)\right]^{1 / 6} .
$$

As before, let us assume that $\Delta^{\prime} a \sim 1 / k a$ for $k \sim$ $k_{\max }$. Then we find from Eq. (11) that $\gamma \propto k^{-2 / 3}$, whereas Eq. (12) yields $\gamma \propto k^{2 / 3}$. Scalings for the fastest growing mode can thus again be found by balancing Eqs. (11) and (12). The result is

$$
\begin{aligned}
k_{\max } a & \sim\left(a / B_{0}\right)^{1 / 4} \tau_{\eta}^{-1 / 8} \tau_{\nu}^{-1 / 8}, \\
\gamma_{\max } & \sim\left(a / B_{0}\right)^{-1 / 2} \tau_{\eta}^{-3 / 4} \tau_{\nu}^{1 / 4}, \\
\delta_{\text {inner }} / a & \sim\left(a / B_{0}\right)^{1 / 4} \tau_{\eta}^{-1 / 8} \tau_{\nu}^{-1 / 8} .
\end{aligned}
$$

We now repeat the previous procedure of rescaling the equilibrium length scale $a$, this time using the results obtained by Park et al. 36] for the SP model in the limit $P m \gg 1$ :

$$
a \equiv \delta_{\mathrm{CS}} \sim L_{\mathrm{CS}} S^{-1 / 2} \mathrm{Pm}^{1 / 4}
$$

This gives

$$
\begin{aligned}
k_{\max } L_{\mathrm{CS}} & \sim S^{3 / 8} P m^{-3 / 16}, \\
\gamma_{\max } \tau_{A} & \sim S^{1 / 4} P m^{-5 / 8}, \\
\delta_{\text {inner }} / \delta_{\mathrm{CS}} & \sim S^{-1 / 8} \mathrm{Pm}^{1 / 16} .
\end{aligned}
$$

We shall find in section VI that these scalings indeed agree very well with the results of a direct numerical integration of the linearized equations. We thus find that the dependence of $\gamma_{\max }$ and $k_{\max }$ on $S$ remains unchanged at large $P m$. However, viscosity damps the instability, and decreases the wave-number and the growth rate of the fastest growing mode, while slightly thickening the inner layer. 
An important question is how the critical Lundquist number for the onset of the current sheet instability, $S_{\text {crit }}$, scales with the magnetic Prandtl number. Although the expressions above are formally only valid in the limit $S \gg S_{\text {crit }}, P m \gg 1$, we can use them to obtain a rough estimate of this dependence. Since the instability requires $\delta_{\text {inner }} / \delta_{\mathrm{CS}} \ll 1$, we may again demand that this be at most 1/3 and use Eq. (20) to obtain

$$
S_{\text {crit }} \sim 10^{4} \mathrm{Pm}^{1 / 2} .
$$

To see the consistency of this result, note that the same dependence of $S_{\text {crit }}$ on $P m$ can be obtained by either looking for the minimum wavenumber that will fit inside the current sheet, $k_{\max } L_{\mathrm{CS}} \sim 1$, or by requiring that the growth rate is comparable to the flow shear rate, $\gamma_{\max } L_{\mathrm{CS}} / u_{\text {out }} \sim 1$ (note that for $P m \gg 1$, $u_{\text {out }} \sim V_{A} P m^{-1 / 2}$ 36]). In both cases, Eq. (18) and Eq. (19) yield $S_{\text {crit }} \sim P m^{1 / 2}$. This result is a specific prediction, which can in principle be checked via direct numerical simulations of the current-sheet instability in the large-Prandtl-number regime.

\section{Kelvin-Helmholtz instability}

The velocity outflow profile of a Sweet-Parker reconnection configuration is such that it is maximum at the midplane $(x=0)$ of the current sheet, and decays to zero away from it. Thus, there are two parallel shear layers, with two corresponding inflection points of the outflow, at $x \sim \pm \delta_{\mathrm{CS}}$. The shear (in the $x$-direction) of this flow profile can be estimated as:

$$
\frac{d u_{y}}{d x} \sim \frac{V_{A}}{\delta_{\mathrm{CS}}} \frac{y}{L_{\mathrm{CS}}} .
$$

Each of these layers would be Kelvin-Helmholtz unstable were it not for the stabilizing effect of the upstream magnetic field, $B_{y}$ [85]: as is well known, a magnetic field that is coplanar with the flow profile will stabilize the $\mathrm{KH}$ instability as long as $\left|B_{y}\right|>\left|u_{y}\right|$. In the case of a SP current sheet, the upstream magnetic field $B_{y}$ is not constant along the sheet; in particular, its magnitude decreases in the $y$-direction; a simple model for it is [86]

$$
B_{y}=B_{0} \sqrt{1-y^{2} / L_{\mathrm{CS}}^{2}} .
$$

It is thus possible that there exists a location $y_{\text {crit }} / L_{\mathrm{CS}} \sim$ 1 along the sheet where the (decreasing) strength of $B_{y}$ matches the (increasing) magnitude of the ouflow, $u_{y} \sim V_{A} y / L_{\mathrm{CS}}$. This is the Alfvén Mach point of the system; for $y>y_{\text {crit }}$, the magnetic field is no longer able to stabilize the KH mode. Fig. 1 provides a schematic illustration of both types (KH stable and unstable) of configuration.

A rigorous derivation of this instability is presented in section $\mathrm{V}$. Here we show how the basic scalings for the fastest growing mode and corresponding wave number

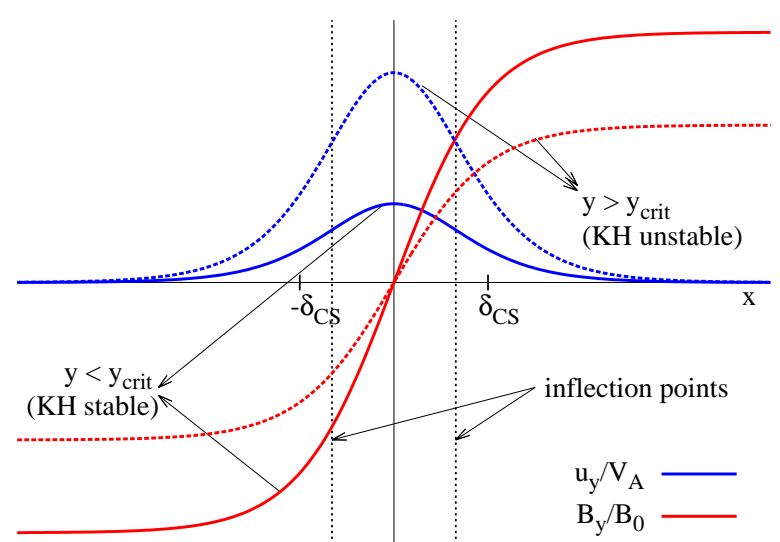

FIG. 1. Cartoon illustrating KH-stable and KH-unstable parts of an idealized SP current sheet. The outflow profile $u_{y}$ is depicted in blue; the upstream magnetic field $B_{y}$ in red. The vertical dashed lines mark the position of KH-unstable layers (i.e., the inflection points of the outflow profile, where $\left.d^{2} u_{y} / d x^{2}=0\right)$. The full lines show the profiles below the Alfvén Mach point $\left(y<y_{\text {crit }}\right)$; in this case the magnitude of $B_{y}$ at $x \sim \pm \delta_{\mathrm{CS}}$ exceeds that of $u_{y}$ at $x=0$, and thus the magnetic field is sufficiently strong to stabilise the current sheet against the KH instability. The opposite case of profiles above the Alfvén Mach point $\left(y>y_{\text {crit }}\right)$ is shown by the dashed lines; $B_{y}$ at the inflow is now weaker than $u_{y}$ at the center of the sheet, which is, therefore, KH-unstable.

can be obtained heuristically. From the standard theory of the KH instability [85], it is known that

$$
\gamma_{\max }^{\mathrm{KH}} \sim \frac{d u_{y}}{d x} \sim \frac{V_{A}}{a}, \quad k_{\max }^{\mathrm{KH}} a \sim 1,
$$

where $a$ is the characteristic scale length of the sheared flow profile $u_{y}(x)$. As above, these estimates can be applied to the SP-sheet by simply setting $a \rightarrow \delta_{\mathrm{CS}} \sim$ $L_{\mathrm{CS}} S^{-1 / 2}$, implying that $k_{\max }^{\mathrm{KH}} \sim 1 / \delta_{\mathrm{CS}}$. Thus,

$$
\gamma_{\max }^{\mathrm{KH}} \tau_{A} \sim S^{1 / 2}, \quad k_{\max }^{\mathrm{KH}} L_{\mathrm{CS}} \sim S^{1 / 2} .
$$

Note that this growth rate is even larger (i.e., has a steeper positive scaling with $S$ ) than that of the plasmoid instability, Eq. (9).

The KH instability scalings in the large $P m$ limit are easily obtained in a similar way, using the following modifications of the SP relations derived in [36]: $u_{\text {out }} \sim V_{A} P m^{-1 / 2}, a \rightarrow \delta_{\mathrm{CS}} \sim L_{\mathrm{CS}} S^{-1 / 2} \mathrm{Pm}^{1 / 4}$. Then we have

$$
\gamma_{\max }^{\mathrm{KH}} \tau_{A} \sim S^{1 / 2} \mathrm{Pm}^{-3 / 4}, \quad k_{\max }^{\mathrm{KH}} L_{\mathrm{CS}} \sim S^{1 / 2} \mathrm{Pm}^{-1 / 4} .
$$

\section{PROBLEM SETUP}

In this section we proceed to make some of the above discussion more rigorous and quantitative. 
We solve the 2D reduced-MHD equations [87,

$$
\begin{aligned}
& \partial_{t} \nabla_{\perp}^{2} \phi+\left\{\phi, \nabla_{\perp}^{2} \phi\right\}=\left\{\psi, \nabla_{\perp}^{2} \psi\right\}+\nu \nabla_{\perp}^{4} \phi, \\
& \partial_{t} \psi+\{\phi, \psi\}=\eta \nabla_{\perp}^{2} \psi-E_{0} .
\end{aligned}
$$

Here $\phi$ and $\psi$ are the stream and flux functions of the in-plane velocity and magnetic field, respectively, so $\boldsymbol{u}=\left(-\partial_{y} \phi, \partial_{x} \phi\right), \boldsymbol{B}=\left(-\partial_{y} \psi, \partial_{x} \psi\right)$; the magnetic field is measured in velocity units; Poisson brackets are denoted by $\{\phi, \psi\}=\partial_{x} \phi \partial_{y} \psi-\partial_{y} \phi \partial_{x} \psi ; \eta$ and $\nu$ denote the plasma resistivity and viscosity, respectively; $E_{0}$ represents an externally applied electric field, required to sustain an equilibrium in the presence of finite resistivity. In the analytical calculation that follows, we will assume that the magnetic Prandtl number is small, $P m=\nu / \eta \ll 1$ and therefore neglect the effect of viscosity on the linear instability. The case of large $P m$ will be studied numerically in section VIB

We are interested in analysing the linear stability of an SP-like current sheet, whose inverse aspect ratio, $\epsilon$, is predicted by the SP model to scale as

$$
\epsilon=\delta_{\mathrm{CS}} / L_{\mathrm{CS}} \sim S^{-1 / 2} \ll 1 .
$$

Therefore, in the vicinity of a general point along the current sheet, $y=y_{0}$, and provided that $y_{0}$ is not too close to either of the ends of the sheet, it is reasonable to expand the equilibrium magnetic flux and stream functions in a power series [88]:

$$
\begin{aligned}
& \left.\psi_{e q}(x, y)\right|_{y=y_{0}}=\sum_{n=0}^{\infty} \frac{1}{n !}\left(\frac{y-y_{0}}{L_{\mathrm{CS}}}\right)^{n} \psi_{n}\left(x, y_{0}\right), \\
& \left.\phi_{e q}(x, y)\right|_{y=y_{0}}=\sum_{n=0}^{\infty} \frac{1}{n !}\left(\frac{y-y_{0}}{L_{\mathrm{CS}}}\right)^{n} \phi_{n}\left(x, y_{0}\right) .
\end{aligned}
$$

The functions $\psi_{n}\left(x, y_{0}\right), \phi_{n}\left(x, y_{0}\right)$ can in principle be found either by substituting these expansions back into Eqs. (27 28) and solving the equilibrium problem order by order in $\left(y-y_{0}\right) / L_{\mathrm{CS}}$, or by Taylor expanding a known equilibrium solution, $\psi_{e q}, \phi_{e q}$ around the point $y=y_{0}$. Neither of these procedures is straighforward: the first option implies either truncating the expansion at some arbitrary order or guessing one of the equilibrium functions [88] to solve the closure problem; the second requires an exact analytical solution. To the best of our knowledge, a 2D analytical solution to this problem that would capture all the essential features of a resistive SPlike current sheet equilibrium has never been derived [89]. Fortunately, however, we shall find in the following sections that, actually deriving the linear instability requires very little information about the equilibrium profiles, and the problem can be solved for general functions $\psi_{n}, \phi_{n}$ provided that the following key assumptions hold:

- the background equilibrium observes the expected symmetries, i.e., at the center of the sheet

$$
\psi_{2 n+1}\left(y_{0}=0\right)=0, \quad \phi_{2 n}\left(y_{0}=0\right)=0 ;
$$

- for $|x| \gg 1$, the incoming flow $u_{x}$ and the reconnecting magnetic field $B_{y}$ approach constant (in $x$ ) values (which can, however, be functions of $y_{0}$ ).

Further general properties of the equilibrium that will be needed in our calculation are derived in Appendix A

Note that, in Paper I, we considered only the case $y_{0}=0$. Furthermore, we adopted a very simplified description of an equilibrium current sheet in which only $\phi_{1}$ and $\psi_{0}$ were non-zero, with $\phi_{1}=\Gamma_{0} x y, \psi_{0}=\psi_{0}(x)$, i.e., the flow had no vorticity $\omega_{z}=\nabla_{\perp}^{2} \phi_{1}=0$, and the reconnected field was ignored, $B_{x}=-\partial \psi / \partial y=0$. In this paper, we drop those model assumptions and consider an arbitrary, two-dimensional current-sheet equilibrium.

\section{A. Normalizations}

We introduce the following normalizations, motivated by the SP scalings:

$$
\begin{array}{ll}
\phi_{0}^{\prime}=\Gamma_{0} y_{0} v(x) ; & \psi_{0}^{\prime}=B_{0} f(x) ; \\
\phi_{1}=-\Gamma_{0} L_{\mathrm{CS}} \delta_{\mathrm{CS}} u(x) ; & \psi_{1}=-\Gamma_{0} y_{0} \delta_{\mathrm{CS}} g(x) ; \\
\phi_{2}=-\Gamma_{0} y_{0} \delta_{\mathrm{CS}} w(x) ; & \psi_{2}=-\Gamma_{0} L_{\mathrm{CS}} \delta_{\mathrm{CS}} h(x) ;
\end{array}
$$

where

$$
\delta_{\mathrm{CS}}=\left(\eta / \Gamma_{0}\right)^{1 / 2}
$$

and $\Gamma_{0}=B_{0} / L_{\mathrm{CS}}$ (note that in our units $B_{0}=V_{A}$ ). We also normalise time and lengths as follows:

$$
t \Gamma_{0}=\tau ; \quad x / \delta_{\mathrm{CS}}=\xi ; \quad y / L_{\mathrm{CS}}=\bar{y}
$$

Under these normalizations, the magnetic and velocity fields obtained from the power-series expansions (30 31) keeping only up to first-order corrections in $\bar{y}-\bar{y}_{0}$ are:

$$
\begin{aligned}
& B_{y} / B_{0}=f(\xi)-\left(\bar{y}-\bar{y}_{0}\right) \bar{y}_{0} g^{\prime}(\xi) \text { (reconnecting) } \\
& B_{x} / B_{0}=\epsilon\left[\bar{y}_{0} g(\xi)+\left(\bar{y}-\bar{y}_{0}\right) h(\xi)\right] \text { (reconnected) }
\end{aligned}
$$

and

$$
\begin{aligned}
& u_{y} / V_{A}=\bar{y}_{0} v(\xi)-\left(\bar{y}-\bar{y}_{0}\right) u^{\prime}(\xi) \text { (outflow) } \\
& u_{x} / V_{A}=\epsilon\left[u(\xi)-\left(\bar{y}-\bar{y}_{0}\right) \bar{y}_{0} w(\xi)\right] \text { (inflow). }
\end{aligned}
$$

It is clear from these expressions what the physical significance of the functions $f, g, h, v, u, w$ is. The physical units and presumed magnitudes of these fields have been absorbed into the normalizations, so these functions are all order-unity dimensionless quantities. They can have parametric dependence on $\bar{y}_{0}$, but note that the presumed lowest-order linear dependence of the reconnected magnetic field and of the outflow on $\bar{y}_{0}$ are explicitly included in the normalizations Eq. (33). 


\section{B. Linearized Equations}

Let us consider small perturbations to a generic equilibrium, $\psi=\psi_{e q}+\delta \psi(x, y, t), \phi=\phi_{e q}+\delta \phi(x, y, t)$, and linearize the RMHD Eqs. (27 28) using the expansions (30 31) for the equilibrium profiles, keeping terms up to first order in $\left(\bar{y}-\bar{y}_{0}\right)$. We obtain

$$
\begin{gathered}
\frac{\partial \delta \psi}{\partial \tau}+\left[\bar{y}_{0} v(\xi)-\left(\bar{y}-\bar{y}_{0}\right) u^{\prime}(\xi)\right] \frac{\partial \delta \psi}{\partial \bar{y}}+\left[u(\xi)+\left(\bar{y}-\bar{y}_{0}\right) \bar{y}_{0} w(\xi)\right] \frac{\partial \delta \psi}{\partial \xi} \\
-\left[\bar{y}_{0} g(\xi)+\left(\bar{y}-\bar{y}_{0}\right) h(\xi)\right] \frac{\partial \delta \phi}{\partial \xi}-\left[f(\xi)-\left(\bar{y}-\bar{y}_{0}\right) \bar{y}_{0} g^{\prime}(\xi)\right] \frac{\partial \delta \phi}{\partial \bar{y}}=\left(\frac{\partial^{2}}{\partial \xi^{2}}+\epsilon^{2} \frac{\partial^{2}}{\partial \bar{y}^{2}}\right) \delta \psi+\mathcal{O}\left(\left(\bar{y}-\bar{y}_{0}\right)^{2}\right), \\
\left\{\frac{\partial}{\partial \tau}+\left[\bar{y}_{0} v(\xi)-\left(\bar{y}-\bar{y}_{0}\right) u^{\prime}(\xi)\right] \frac{\partial}{\partial \bar{y}}+\left[u(\xi)+\left(\bar{y}-\bar{y}_{0}\right) \bar{y}_{0} w(\xi)\right] \frac{\partial}{\partial \xi}\right\}\left(\frac{\partial^{2}}{\partial \xi^{2}}+\epsilon^{2} \frac{\partial^{2}}{\partial \bar{y}^{2}}\right) \delta \phi \\
-\left[u^{\prime \prime}(\xi)+\left(\bar{y}-\bar{y}_{0}\right) \bar{y}_{0} w^{\prime \prime}(\xi)\right] \frac{\partial \delta \phi}{\partial \xi}-\left[\bar{y}_{0} v^{\prime \prime}(\xi)-\left(\bar{y}-\bar{y}_{0}\right) u^{\prime \prime \prime}(\xi)\right] \frac{\partial \delta \phi}{\partial \bar{y}}= \\
\left\{\left[f(\xi)-\left(\bar{y}-\bar{y}_{0}\right) \bar{y}_{0} g^{\prime}(\xi)\right] \frac{\partial}{\partial \bar{y}}+\left[\bar{y}_{0} g(\xi)+\left(\bar{y}-\bar{y}_{0}\right) h(\xi)\right] \frac{\partial}{\partial \xi}\right\}\left(\frac{\partial^{2}}{\partial \xi^{2}}+\epsilon^{2} \frac{\partial^{2}}{\partial \bar{y}^{2}}\right) \delta \psi \\
-\left[\bar{y}_{0} g^{\prime \prime}(\xi)+\left(\bar{y}-\bar{y}_{0}\right) h^{\prime \prime}(\xi)\right] \frac{\partial \delta \psi}{\partial \xi}-\left[f^{\prime \prime}(\xi)+\left(\bar{y}-\bar{y}_{0}\right) \bar{y}_{0} g^{\prime \prime}\right] \frac{\partial \delta \psi}{\partial \bar{y}}+\mathcal{O}\left(\left(\bar{y}-\bar{y}_{0}\right)^{2}\right),
\end{gathered}
$$

where we have used the normalizations defined in Eqs. (33) 35).

In the case of $y_{0}=0$, the above equations include the following effects which were absent in Paper I:

- in Eq. (40), the term proportional to $h(\xi)$, represents the effect of the reconnected magnetic field;

- in Eq. (41), the terms proportional to $u^{\prime \prime}(\xi)$ and $u^{\prime \prime \prime}(\xi)$ on the left-hand side, which represent the vorticity of the equilibrium flow;

- the term proportional to $h^{\prime \prime}(\xi)$ on the right-hand side of Eq. (41), which is the contribution to the equilibrium-current gradient from the reconnected magnetic field.

Further progress at this point is hindered by the fact that these equations contain explicit dependences on the $y$ variable and cannot, therefore, be Fourier transformed in this direction. To address this difficulty, let us compare the magnitudes of the first and third terms on the lefthand sides of these equations:

$$
\frac{\partial / \partial \tau}{\left(\bar{y}-\bar{y}_{0}\right) \partial / \partial \bar{y}} \sim \frac{\gamma}{\left(\bar{y}-\bar{y}_{0}\right) \kappa},
$$

where $\gamma$ is the growth rate of the anticipated instability at $\bar{y}=\bar{y}_{0}$ normalized to the Alfvénic shearing rate $\Gamma_{0}$ and

$$
\kappa=k L_{\mathrm{CS}}
$$

is the normalized wavenumber of the perturbation at that location. Thus, the third term can be ignored if the analysis is restricted to patches of the current sheet whose extent in the $y$-direction is such that

$$
\left(\bar{y}-\bar{y}_{0}\right) \ll \gamma / \kappa .
$$

This approach is valid provided that

$$
\kappa\left(\bar{y}-\bar{y}_{0}\right) \gg 1 .
$$

In other words, the domain in the $y$-direction is divided into smaller patches, and the linear analysis performed locally in of each of these. A WKB approach remains valid provided that asymptotically many wave lengths fit in each of these patches. Eqs. (44, 45) imply that we seek solutions such that

$$
\frac{\partial}{\partial \tau} \sim \gamma_{\max } \gg 1, \quad \frac{\partial}{\partial \bar{y}} \sim \kappa_{\max } \gg 1 .
$$

These are a priori assumptions, which will be later justified by our ability to obtain such solutions.

Under these approximations, Eqs. (40 41) become 


$$
\begin{aligned}
& \frac{\partial \delta \psi}{\partial \tau}+\bar{y}_{0} v(\xi) \frac{\partial \delta \psi}{\partial \bar{y}}+u(\xi) \frac{\partial \delta \psi}{\partial \xi}-\bar{y}_{0} g(\xi) \frac{\partial \delta \phi}{\partial \xi}-f(\xi) \frac{\partial \delta \phi}{\partial \bar{y}}=\left(\frac{\partial^{2}}{\partial \xi^{2}}+\epsilon^{2} \frac{\partial^{2}}{\partial \bar{y}^{2}}\right) \delta \psi \\
& \left\{\frac{\partial}{\partial \tau}+\bar{y}_{0} v(\xi) \frac{\partial}{\partial \bar{y}}+u(\xi) \frac{\partial}{\partial \xi}\right\}\left(\frac{\partial^{2}}{\partial \xi^{2}}+\epsilon^{2} \frac{\partial^{2}}{\partial \bar{y}^{2}}\right) \delta \phi-u^{\prime \prime}(\xi) \frac{\partial \delta \phi}{\partial \xi}-\bar{y}_{0} v^{\prime \prime}(\xi) \frac{\partial \delta \phi}{\partial \bar{y}}= \\
& {\left[f(\xi) \frac{\partial}{\partial \bar{y}}+\bar{y}_{0} g(\xi) \frac{\partial}{\partial \xi}\right]\left(\frac{\partial^{2}}{\partial \xi^{2}}+\epsilon^{2} \frac{\partial^{2}}{\partial \bar{y}^{2}}\right) \delta \psi-\bar{y}_{0} g^{\prime \prime}(\xi) \frac{\partial \delta \psi}{\partial \xi}-f^{\prime \prime}(\xi) \frac{\partial \delta \psi}{\partial \bar{y}} .}
\end{aligned}
$$

Note that the functions $h(\xi)$ and $w(\xi)$ have dropped out.

One can now look for linear modes of the form $\exp (-i \omega \tau)$. In the midplane of the current sheet (i.e., $\xi=0)$, the plasma is flowing outwards at some fraction of the Alfvén speed, $\bar{y}_{0} v_{0}$, where $v_{0}=v\left(\xi=0, \bar{y}_{0}\right)$. Let us take this into consideration explicitly and set

$$
\omega=\kappa \bar{y}_{0} v_{0}+i \gamma .
$$

We therefore look for solutions to Eqs. (47 48) in the form:

$$
\begin{aligned}
\delta \psi & =\Psi(\xi) e^{\left[\gamma-i \kappa \bar{y}_{0} v_{0}\right] \tau+i \kappa \bar{y}}, \\
\delta \phi & =-i \Phi(\xi) e^{\left[\gamma-i \kappa \bar{y}_{0} v_{0}\right] \tau+i \kappa \bar{y}} .
\end{aligned}
$$

We are ignoring the time dependence of $\kappa$ due to the background flows, because this variation will occur on a much longer timescale than that of the expected growth rate of the instability [90]. Introducing the parameter

$$
\lambda=\gamma / \kappa,
$$

we obtain

$$
\begin{aligned}
& \left\{\lambda-i \bar{y}_{0}\left[v_{0}-v(\xi)\right]\right\} \Psi+\frac{u(\xi)}{\kappa} \Psi^{\prime} \\
& +i \frac{\bar{y}_{0}}{\kappa} g(\xi) \Phi^{\prime}-f(\xi) \Phi=\frac{1}{\kappa}\left(\Psi^{\prime \prime}-\kappa^{2} \epsilon^{2} \Psi\right) \\
& \left\{\lambda-i \bar{y}_{0}\left[v_{0}-v(\xi)\right]\right\}\left(\Phi^{\prime \prime}-\kappa^{2} \epsilon^{2} \Phi\right) \\
& +\frac{u(\xi)}{\kappa}\left(\Phi^{\prime \prime \prime}-\kappa^{2} \epsilon^{2} \Phi^{\prime}\right)-\frac{u^{\prime \prime}(\xi)}{\kappa} \Phi^{\prime}-i \bar{y}_{0} v^{\prime \prime}(\xi) \Phi= \\
& -f(\xi)\left(\Psi^{\prime \prime}-\kappa^{2} \epsilon^{2} \Psi\right)+i \frac{\bar{y}_{0}}{\kappa} g(\xi)\left(\Psi^{\prime \prime \prime}-\kappa^{2} \epsilon^{2} \Psi^{\prime}\right) \\
& \quad-i \frac{\bar{y}_{0}}{\kappa} g^{\prime \prime}(\xi) \Psi^{\prime}+f^{\prime \prime}(\xi) \Psi .
\end{aligned}
$$

This is the set of equations that will be solved in sections IV and V

\section{PLASMOID INSTABILITY}

We will now proceed to solve Eqs. (53) 54 in three different regions: the "external" (global) region where
$\delta_{\mathrm{CS}} \ll x \ll L$ (i.e., $\left.1 \ll \xi \ll S^{1 / 2}\right)$, the "outer" region (the SP current sheet), where $x \sim \delta_{\mathrm{CS}}(\xi \sim 1)$ and finally the "inner" region (the inner layer inside the current sheet where plasmoids form), where $x \ll \delta_{\mathrm{CS}}(\xi \ll 1)$. Outside the current sheet (i.e., in the external region), the plasma is ideal; inside the current sheet (i.e., in the outer and inner regions), resistive effects cannot be neglected. In addition to the assumptions of Eq. (46), we will require here that $\lambda=\gamma / \kappa \ll 1$. This ordering is indeed satisfied by the fastest growing mode, as we will confirm a posteriori.

\section{A. External Region: $|\xi| \gg 1$}

This is the upstream region outside the current layer, i.e., $x \gg \delta_{\mathrm{CS}}$. Here, we expect the equilibrium profiles to behave as:

$$
\begin{array}{lll}
v(\xi) \rightarrow 0, & u(\xi) \approx \mp u_{\infty}, \\
f(\xi) \approx \pm f_{\infty}, & g(\xi) \approx \pm g_{\infty}^{\prime} \xi,
\end{array}
$$

where $u_{\infty}, f_{\infty}, g_{\infty}^{\prime}$ are functions of $\bar{y}_{0}$ only, taken to be of order unity (see Appendix $\mathrm{A}$ ); of these, we will discover that only $f_{\infty}$ matters for the calculation of the instability. In the above expressions, the upper sign applies to $\xi>0$, and the lower sign to $\xi<0$ (so as to observe the expected parities of the equilibrium, namely that $u(\xi)$ and $f(\xi)$ are odd in $\xi$, and $g(\xi)$ is even). The linear dependence of $g(\xi)$ on $\xi$ for large $\xi$ might not be obvious at first glance and is derived in Appendix $\mathrm{A}$.

We will make the a priori assumption that the terms proportional to $u(\xi)$ or $u^{\prime \prime}(\xi)$ and to $g(\xi)$ or $g^{\prime \prime}(\xi)$ in Eqs. (53 54) are negligible in this region, and then show that this is indeed the case. In the absence of these terms, we obtain:

$$
\begin{aligned}
\left(\lambda-i \bar{y}_{0} v_{0}\right) \Psi & = \pm f_{\infty} \Phi, \\
\left(\lambda-i \bar{y}_{0} v_{0}\right)\left(\Phi^{\prime \prime}-\kappa^{2} \epsilon^{2} \Phi\right) & =\mp f_{\infty}\left(\Psi^{\prime \prime}-\kappa^{2} \epsilon^{2} \Psi\right),
\end{aligned}
$$

which can be easily combined to yield:

$$
\left[f_{\infty}^{2}-\left(\lambda-i \bar{y}_{0} v_{0}\right)^{2}\right]\left(\Psi^{\prime \prime}-\kappa^{2} \epsilon^{2} \Psi\right)=0 .
$$

The general solution to this equation is simply

$$
\Psi^{ \pm}=C_{3}^{ \pm} e^{\mp \kappa \epsilon \xi},
$$


where $C_{3}^{ \pm}$are integration constants and \pm refers to $\xi \gtrless 0$ [in unscaled units: $\Psi^{ \pm}=C_{3}^{ \pm} \exp (\mp k x)$ ].

We can now check the assumption about the smallness of the terms proportional to $u(\xi), u^{\prime \prime}(\xi), g(\xi)$ and $g^{\prime \prime}(\xi)$. From Eq. (60) and Eq. (57) we see that, for arbitrary $y_{0}$, we have $\Psi^{\prime} / \Psi \sim \Phi^{\prime} / \Phi \sim \kappa \epsilon$. Let us then compare the magnitudes of the third and first terms on the left-hand side of Eq. (53) [the same reasoning applies to Eq. (54)]:

$$
\frac{\left(u_{\infty} / \kappa\right) \Psi^{\prime}}{\lambda \Psi} \sim \lambda^{-1} \epsilon
$$

which is small provided that $\lambda \gg \epsilon$, a condition we will later see is satisfied by the fastest growing mode. With respect to terms involving $g(\xi)$, the ratio of the magnitudes of the fourth and fifth terms on the left-hand side of Eq. (53) [and similarly for the second and first terms on the right-hand side of Eq. (54)] is

$$
\bar{y}_{0} \frac{\xi}{f_{\infty}^{2} \kappa} \frac{\Phi^{\prime}}{\Phi} \sim \xi \epsilon
$$

This is again small provided that $\xi \ll \epsilon^{-1} \sim S^{-1 / 2}$; we shall find that the fastest growing wave number $\kappa_{\max } \sim$ $\epsilon^{-3 / 4}$ and thus the eigenfunction, Eq. (60), decays before the condition $\xi \epsilon \ll 1$ breaks down. Note also that the expression for $g(\xi)$ given in Eq. (56) is not expected to hold for $\xi>\epsilon^{-1}$ (i.e., $x>L_{\mathrm{CS}}$ ): the reconnected magnetic field does not grow unbounded as $\xi \rightarrow \infty)$.

\section{B. Outer Region: $|\xi| \sim 1$}

This region represents the SP current sheet itself, i.e., $x \sim \delta_{\mathrm{CS}}$. Here, the functions $u(\xi), v(\xi), f(\xi)$ and $g(\xi)$ are simply assumed to be $\sim \mathcal{O}(1)$. We find that terms proportional to $u(\xi)$ and $g(\xi)$ or to their derivatives are again negligible. For example, consideration of the same terms as in the previous section leads to:

$$
\begin{gathered}
\frac{u \Psi^{\prime} / \kappa}{\lambda \Psi} \sim \gamma^{-1} \ll 1 \\
\frac{\bar{y}_{0} g \Phi^{\prime} / \kappa}{f \Phi} \sim \bar{y}_{0} \kappa^{-1} \ll 1
\end{gathered}
$$

and similarly for the others. Therefore, to lowest order in $\epsilon$, Eqs. (53 54) become

$$
\left\{\lambda-i \bar{y}_{0}\left[v_{0}-v(\xi)\right]\right\} \Psi=f(\xi) \Phi,
$$

$$
\begin{gathered}
\left\{\lambda-i \bar{y}_{0}\left[v_{0}-v(\xi)\right]\right\}\left(\Phi^{\prime \prime}-\kappa^{2} \epsilon^{2} \Phi\right)-i \bar{y}_{0} v^{\prime \prime}(\xi) \Phi= \\
-f(\xi) \Psi^{\prime \prime}+\left[f^{\prime \prime}(\xi)+\kappa^{2} \epsilon^{2} f(\xi)\right] \Psi .
\end{gathered}
$$

Combining these equations results in the following eigenvalue problem:

$$
\Psi^{\prime \prime}-\left[\frac{f^{\prime \prime}(\xi)}{f(\xi)}+\kappa^{2} \epsilon^{2}\right] \Psi=-\frac{\lambda-i \bar{y}_{0}\left[v_{0}-v(\xi)\right]}{f(\xi)}\left\{\left(\frac{d^{2}}{d \xi^{2}}-\kappa^{2} \epsilon^{2}\right) \frac{\lambda-i \bar{y}_{0}\left[v_{0}-v(\xi)\right]}{f(\xi)} \Psi-\frac{i \bar{y}_{0} v^{\prime \prime}(\xi)}{f(\xi)} \Psi\right\}
$$

subject to boundary conditions given by the external solution, Eq. (60), and the requirement (for the plasmoid instability) that $\Psi$ be an even function. Writing the solution in the form $\Psi(\xi)=f(\xi) \chi(\xi)$, Eq. (67) becomes:

$$
\frac{d}{d \xi}\left[V(\xi) \chi^{\prime}(\xi)\right]=\epsilon^{2} \kappa^{2} V(\xi) \chi(\xi),
$$

where the "potential" $V(\xi)$ is

$$
V(\xi)=f^{2}(\xi)+\left[\lambda-i y_{0}\left(v_{0}-v(\xi)\right)\right]^{2} .
$$

Note that in the case $y_{0} \ll 1$ all terms on the righthand side of Eq. [67) are small compared to the first two terms on the left-hand side. In that case, we recover to the problem solved in Paper I [except here $f(\xi)$ remains unspecified]. For the general case $\bar{y}_{0} \sim 1$, an exact solution can be obtained provided that the terms proportional to $\kappa^{2} \epsilon^{2}$ can be neglected (we will later check the validity of this assumption; for now, let us call attention to what it means: $\kappa \epsilon=k \delta_{\mathrm{CS}}$, so the assumption $\kappa \epsilon \ll 1$ implies that the wavelength of the expected instability is much longer than the current sheet thickness). Thus, we neglect the right-hand side of Eq. (68) and find the solution

$$
\Psi^{ \pm}(\xi)=C_{1}^{ \pm} f(\xi)+C_{2}^{ \pm} f(\xi) \int_{\xi_{0}}^{\xi} \frac{d \xi^{\prime}}{V\left(\xi^{\prime}\right)} .
$$

where \pm refers to $\xi \gtrless 0, C_{1}^{ \pm}, C_{2}^{ \pm}$are constants in integration and $\xi_{0}$ is an arbitrary number of order unity (different choices of $\xi_{0}$ will produce subdominant corrections to $C_{1}^{ \pm}$).

For the plasmoid instability, we expect $\lambda \ll 1$, so this solution simplifies to

$$
\begin{aligned}
\Psi^{ \pm}= & C_{1}^{ \pm} f(\xi)+ \\
& C_{2}^{ \pm} f(\xi) \int_{\xi_{0}}^{\xi} \frac{d \xi^{\prime}}{f^{2}\left(\xi^{\prime}\right)-\bar{y}_{0}^{2}\left[v_{0}-v\left(\xi^{\prime}\right)\right]^{2}} .
\end{aligned}
$$

We now match this expression to the external solution, Eq. (60), in the region $1 \ll \xi \ll(\kappa \epsilon)^{-1}$, or, equivalently, in dimensional form, $\delta_{C S} \ll x \ll 1 / k$. In this region, $v( \pm \xi) \ll v_{0}$ and $f^{\prime}( \pm \xi) \ll 1$, implying that $|f( \pm \xi)| \approx f_{\infty}$ and the integral is dominated by the upper limit. We 
obtain

$$
\begin{aligned}
& C_{3}^{ \pm}=-\frac{C_{2}^{ \pm} f_{\infty}}{f_{\infty}^{2}-\bar{y}_{0}^{2} v_{0}^{2}} \frac{1}{\kappa \epsilon}, \\
& C_{1}^{ \pm}=\mp \frac{C_{2}^{ \pm}}{f_{\infty}^{2}-\bar{y}_{0}^{2} v_{0}^{2}} \frac{1}{\kappa \epsilon} .
\end{aligned}
$$

For $\xi \ll 1, f(\xi) \approx f_{0}^{\prime} \xi$ and $v(\xi)=v_{0}-v_{0}^{\prime \prime} \xi^{2} / 2$. Thus, to lowest order in $\xi$, the integrand in Eq. (71) becomes $1 /\left(f_{0}^{\prime 2} \xi^{2}\right)$. The integral is therefore again dominated by the upper limit and we obtain:

$$
\Psi^{ \pm}(0)=-\frac{C_{2}^{ \pm}}{f_{0}^{\prime}}
$$

Demanding that $\Psi$ be an even function of $\xi$ yields

$$
C_{2}^{+}=C_{2}^{-}=-f_{0}^{\prime} \Psi(0) .
$$

Thus, the outer region solution is:

$$
\begin{aligned}
\Psi^{ \pm}(\xi)=f_{0}^{\prime} & \Psi(0) f(\xi)\left\{ \pm \frac{1}{f_{\infty}^{2}-\bar{y}_{0}^{2} v_{0}^{2}} \frac{1}{\kappa \epsilon}\right. \\
& \left.-\int_{\xi_{0}}^{\xi} \frac{d \xi^{\prime}}{f^{2}\left(\xi^{\prime}\right)-\bar{y}_{0}^{2}\left[v_{0}-v\left(\xi^{\prime}\right)\right]^{2}}\right\} .
\end{aligned}
$$

Note that the second term of the above expression ensures that the eigenfunction remains finite at $\xi=0$.

As usual in tearing-mode-type calculations, let us now introduce the standard instability parameter

$$
\Delta^{\prime}=\frac{\Psi^{\prime}(+0)-\Psi^{\prime}(-0)}{\Psi(0)} .
$$

Using Eq. (76), we obtain 91]

$$
\Delta^{\prime}=\frac{2}{\kappa \epsilon} \frac{f_{0}^{\prime 2}}{f_{\infty}^{2}-\bar{y}_{0}^{2} v_{0}^{2}} .
$$

We stress that the functional dependence of $\Delta^{\prime}$ on $\bar{y}_{0}$ is both explicit and implicit, as $f_{0}^{\prime}, f_{\infty}$ and $v_{0}$ are all functions of $\bar{y}_{0}$.

\section{Inner Region: $|\xi| \ll 1$}

This region is the internal layer inside the SP current sheet, i.e., $x \ll \delta_{\mathrm{CS}}$. We begin by noting that, again, independently of the specific functional form of the SP equilibrium, the symmetries of the problem are such that, for $|\xi| \ll 1$, the equilibrium profiles can be approximated as

$$
\begin{array}{ll}
f(\xi)=f_{0}^{\prime} \xi+\mathcal{O}\left(\xi^{3}\right), & g(\xi)=g_{0}+\mathcal{O}\left(\xi^{2}\right), \\
u(\xi)=u_{0}^{\prime} \xi+\mathcal{O}\left(\xi^{3}\right), & v(\xi)=v_{0}+\mathcal{O}\left(\xi^{2}\right),
\end{array}
$$

where $f_{0}^{\prime}, u_{0}^{\prime}, g_{0}, v_{0}$ are constants with respect to $\xi$ but depend on $\bar{y}_{0}$. In this region, the relative magnitudes of the different terms in Eqs. (53) 544) can be reduced to one of the following cases:

$$
\begin{aligned}
\frac{u \Psi^{\prime}}{\kappa} \frac{1}{\lambda \Psi} & \sim \frac{u_{0}^{\prime} \delta_{\text {inner }} \Psi}{\kappa \delta_{\text {inner }}} \frac{\kappa}{\gamma \Psi} \sim \frac{1}{\gamma}, \\
\frac{\bar{y}_{0} g \Phi^{\prime}}{\kappa} \frac{1}{f \Phi} & \sim \frac{g_{0}}{\kappa f_{0}^{\prime} \delta_{\text {inner }}^{2}} \sim \frac{1}{\kappa \delta_{\text {inner }}^{2}}, \\
\frac{\Psi^{\prime \prime}}{\kappa} \frac{1}{\lambda \Psi} & \sim \frac{1}{\gamma \delta_{\text {inner }}^{2}}, \\
\frac{u^{\prime \prime} \Phi^{\prime}}{\kappa} \frac{1}{\lambda \Phi^{\prime \prime}} & \sim \frac{\delta_{\text {inner }}^{2}}{\gamma}, \\
\frac{\bar{y}_{0} v^{\prime \prime} \Phi}{\lambda \Phi^{\prime \prime}} & \sim \frac{\kappa \delta_{\text {inner }}^{2}}{\gamma}, \\
\frac{\bar{y}_{0} g^{\prime \prime} \Psi^{\prime}}{\kappa} \frac{1}{f \Psi^{\prime \prime}} & \sim \frac{1}{\kappa} .
\end{aligned}
$$

Except for Eq. (83), all these ratios can be shown a posteriori to be small. Thus, to lowest order, Eqs. (53 54) become

$$
\begin{aligned}
\lambda \Psi-f_{0}^{\prime} \xi \Phi & =\frac{1}{\kappa} \Psi^{\prime \prime}, \\
\lambda \Phi^{\prime \prime} & =-f_{0}^{\prime} \xi \Psi^{\prime \prime} .
\end{aligned}
$$

These equations are mathematically the same as the equations for the tearing mode in the inner region, except that here the role of the small parameter is played by $1 / \kappa$ rather than resistivity. Since $\Delta^{\prime} \delta_{\text {inner }}$ is not expected to be small, the constant- $\Psi$ approximation [8] cannot be used. Instead, this eigenvalue problem is mathematically equivalent to the one solved by Coppi et al. for the resistive internal kink mode [81]. The resulting dispersion relation is

$$
\begin{array}{r}
-\frac{\pi}{8}\left(\kappa f_{0}^{\prime}\right)^{1 / 3} \Lambda^{5 / 4} \frac{\Gamma\left[\left(\Lambda^{3 / 2}-1\right) / 4\right]}{\Gamma\left[\left(\Lambda^{3 / 2}+5\right) / 4\right]}=\Delta^{\prime}= \\
\frac{2}{\kappa \epsilon} \frac{f_{0}^{\prime 2}}{f_{\infty}^{2}-\bar{y}_{0}^{2} v_{0}^{2}},
\end{array}
$$

where $\Gamma$ is the gamma function and $\Lambda=\gamma f_{0}^{\prime-2 / 3} \kappa^{-2 / 3}$. The width of the inner region is

$$
\delta_{\text {inner }}=\gamma^{1 / 4} \kappa^{-1 / 2} f_{0}^{\prime-1 / 2},
$$

which can be used to confirm the smallness of the terms neglected in deriving Eqs. (87,88).

\section{Solution of the Dispersion Relation, Eq. (89)}

The dispersion relation, Eq. (89), has two relevant limits. For $\Lambda \ll 1$,

$$
\begin{aligned}
\gamma & \approx\left[-\frac{16}{\pi} \frac{\Gamma(5 / 4)}{\Gamma(-1 / 4)}\right]^{4 / 5} \frac{f_{0}^{\prime 2}}{\left(f_{\infty}^{2}-\bar{y}_{0}^{2} v_{0}^{2}\right)^{4 / 5}} \kappa^{-2 / 5} \epsilon^{-4 / 5} \\
& \approx 0.95 \frac{f_{0}^{\prime 2}}{\left(f_{\infty}^{2}-\bar{y}_{0}^{2} v_{0}^{2}\right)^{4 / 5}} \kappa^{-2 / 5} \epsilon^{-4 / 5} .
\end{aligned}
$$


This is valid provided that $\kappa \gg \epsilon^{-3 / 4}$ (but also $\kappa \epsilon \ll 1$, as required by our earlier assumptions).

On the other hand, taking $\Lambda \rightarrow 1-$ (from below), we obtain

$$
\gamma=\left(f_{0}^{\prime} \kappa\right)^{2 / 3}-\frac{\sqrt{\pi}}{3} \frac{f_{\infty}^{2}-\bar{y}_{0}^{2} v_{0}^{2}}{f_{0}^{\prime}} \kappa^{2} \epsilon,
$$

valid for $\kappa \lesssim \epsilon^{-3 / 4}$. The scaling of the fastest growing wave number can be determined by balancing the two terms on the right-hand side of the above expression. The exact result can be obtained by solving the equation $d \gamma / d \kappa=0$ in the limit $\Lambda \rightarrow 1-$. We obtain

$$
\begin{aligned}
\kappa_{\max } & =\left(\frac{1}{\sqrt{\pi}} \frac{f_{0}^{\prime 5 / 3}}{f_{\infty}^{2}-\bar{y}_{0}^{2} v_{0}^{2}}\right)^{3 / 4} \epsilon^{-3 / 4}, \\
\gamma_{\max } & =\frac{2}{3 \pi^{1 / 4}} \sqrt{\frac{f_{0}^{\prime 3}}{f_{\infty}^{2}-\bar{y}_{0}^{2} v_{0}^{2}}} \epsilon^{-1 / 2} .
\end{aligned}
$$

The scalings with $\epsilon$ are the same as those derived in Paper I, although here they have been obtained for a general SP equilibrium. In other words, this linear theory predicts that current sheets are unstable to a superAlfvénic instability whose growth rate increases with the Lundquist number, $\gamma_{\max } \sim S^{1 / 4}$ [recall that $\epsilon=S^{-1 / 2}$; see Eq. (29)]. A plasmoid chain forms inside a region of width $\delta_{\text {inner }} \sim S^{-1 / 8} \delta_{\mathrm{CS}}$, with the number of plasmoids scaling as $\kappa_{\max } \sim S^{3 / 8}$. These scalings justify the ordering assumptions employed in deriving these results, i.e., $\gamma_{\max } \gg 1, \kappa_{\max } \gg 1$ and $\lambda_{\max } \sim \gamma_{\max } / \kappa_{\max } \sim \epsilon^{1 / 4}$, so, $\epsilon \ll \lambda_{\max } \ll 1$.

Let us now analyze the dependence of $\gamma_{\max }$ and $\kappa_{\max }$ on the position $\bar{y}_{0}$ along the sheet. Note first that the instability vanishes at the locations where the equilibrium current $f_{0}^{\prime}=0$ - the end points of the SP current sheet. Note also that since we have dropped corrections of or$\operatorname{der} \epsilon^{1 / 2}$ in our derivation [for example, the terms proportional to $\kappa^{2} \epsilon^{2}$ in Eq. (68)], the terms proportional to $\bar{y}_{0}^{2}$ in the above expressions are only to be kept if $\bar{y}_{0} \gg \epsilon^{1 / 4}$. For values of $\bar{y}_{0} \lesssim \epsilon^{1 / 4}$, all $\bar{y}_{0}$ corrections are negligible to lowest order and Eqs. (93 94) simplify to yield

$$
\begin{aligned}
& \kappa_{\max }=\pi^{-3 / 8} \bar{E}_{0}^{5 / 4} \epsilon^{-3 / 4}, \\
& \gamma_{\max }=\frac{2}{3 \pi^{1 / 4}} \bar{E}_{0}^{3 / 2} \epsilon^{-1 / 2},
\end{aligned}
$$

where $\bar{E}_{0}=L_{\mathrm{CS}} E_{0} /\left(B_{0}^{2} \delta_{\mathrm{CS}}\right)$ is the normalized background electric field, and we have used the relationship $f_{0}^{\prime}=\bar{E}_{0}$, which follows from Eq. (A3) in the limit $\bar{y}_{0} \lesssim \epsilon^{1 / 4}$ [note that $\left.f_{\infty}\left(\bar{y}_{0}=0\right)=1\right]$.

In the opposite case of $\bar{y}_{0} \gg \epsilon^{1 / 4}$, the dependence of $\kappa_{\max }$ and $\gamma_{\max }$ on $\bar{y}_{0}$ is a nontrivial function of the specific values of the equilibrium coefficients, which are all functions of $\bar{y}_{0}$ (this does not affect the scaling of $\kappa_{\max }$ and $\gamma_{\max }$ with $\epsilon$ ). For $\bar{y}_{0} \ll 1$, exact values of the coeficients of the Taylor expansion of the equilibrium around $\xi=0$ were derived semi-analytically by Uzdensky and
Kulsrud 92] assuming a Syrovatskii-like upstream magnetic field [Eq. (23)] — see Appendix A. Using those coefficients and the relationship $f_{0}^{\prime}=\bar{E}_{0}-\bar{y}_{0}^{2} v_{0} g_{0}$ derived in Appendix A, a Taylor expansion of Eqs. (93 94 in $\bar{y}_{0}$ yields:

$$
\begin{aligned}
& \kappa_{\max }\left(\left|\bar{y}_{0}\right| \ll 1\right) \approx\left(0.56+0.10 \bar{y}_{0}^{2}\right) \epsilon^{-3 / 4}, \\
& \gamma_{\max }\left(\left|\bar{y}_{0}\right| \ll 1\right) \approx\left(0.71+0.73 \bar{y}_{0}^{2}\right) \epsilon^{-1 / 2} .
\end{aligned}
$$

These results reveal a perhaps unexpected feature of the plasmoid instability: both $\kappa_{\max }$ and $\gamma_{\max }$ increase with distance from the center of the sheet. The same conclusion is easily deduced for arbitrary $\bar{y}_{0} \sim 1$ : even though in that case one is forced to retain the full expressions for $\kappa_{\max }$ and $\gamma_{\max }$, Eqs. (93 94), it is also true that, under very general conditions, one expects $f_{\infty}$ to be a decreasing function of $\bar{y}_{0}$ (for example, a standard Syrovatskii ideal current sheet solution [86] yields $f_{\infty}=\sqrt{1-\bar{y}_{0}^{2}}$; see Appendix $\mathrm{A}$.

A problem thus arises: it is possible that a location $\bar{y}_{0}=\bar{y}_{0, \text { crit }} \sim \mathcal{O}(1)$ exists inside the current sheet where $f_{\infty}^{2}-\bar{y}_{0, \text { crit }}^{2} v_{0}^{2}=0$, and our solution breaks down $\left(\Delta^{\prime}, \gamma, \kappa \rightarrow+\infty\right)$. It is clear that while approaching that point, $\kappa_{\max }$ will get to be so large that terms of order $\kappa_{\max } \epsilon$, or $\kappa_{\max }^{2} \epsilon^{2}$, can no longer be neglected and thus our ordering assumptions become invalid. For values of $\left|\bar{y}_{0}\right|>\bar{y}_{0, \text { crit }}$, our solution is again physical, but the second term on the right-hand side of Eq. (92) equation changes sign, implying that $\gamma$ grows with $\kappa$ and the value of the fastest-growing wave number cannot be deduced from this equation. This means that terms proportional to $\kappa^{2} \epsilon^{2}$ are still necessary to determine the fastest growing mode. Physically, the fact that the condition $\kappa_{\max } \epsilon \sim 1$ is met somewhere in the sheet means that at those locations the wavelength of the perturbation becomes comparable to the current sheet thickess, whereas for $\left|\bar{y}_{0}\right| \ll \bar{y}_{0, \text { crit }}$ it was much longer.

It is easy to understand why the location $f_{\infty}^{2}-$ $\bar{y}_{0, \text { crit }}^{2} v_{0}^{2}=0$ should be special: this is where the midplane outflow speed (i.e., $u_{y}$ measured at $x=0$ ) matches the Alfvén velocity associated with the upstream magnetic field (measured at $x \sim \delta_{\mathrm{CS}}$ ), i.e., it is the Alfvén Mach point of the system. The background outflow velocity profile is strongly sheared (in the $x$-direction) inside the current sheet. Such a profile would be unstable to the Kelvin-Helmholtz (KH) instability, were it not for the stabilizing effect provided by the (flow-aligned) background magnetic field $B_{y}$ [85]. However, whereas the flow grows in magnitude with increasing $y, B_{y}$ does the opposite (see the discussion of section IIC). The Alfvén Mach point is where the two amplitudes match. Beyond that point, the magnetic field is no longer sufficiently strong to provide stability, and we should thus expect the plasmoid instability to morph into the KH instability. The increase of $\gamma_{\max }$ and $\kappa_{\max }$ along the sheet is thus a reflection of the fact that the current sheet is increasingly unstable to the $\mathrm{KH}$ mode. For $\bar{y}_{0} \gtrsim \bar{y}_{0, \text { crit }}$ the plasmoid instability is replaced by the $\mathrm{KH}$ instability as the most unstable 
mode. The most unstable wave number also grows along the sheet because, as is well known [85], the growth rate of the $\mathrm{KH}$ instability peaks at $\kappa \epsilon \sim 1$ (i.e., $k \delta_{\mathrm{CS}} \sim 1$, where $\delta_{\mathrm{CS}}$ is the scale of the cross-sheet velocity shear).

To summarize, the analytical dispersion relation, Eq. [89], accurately describes the plasmoid instability of the current sheet for $-\bar{y}_{0, \text { crit }}<\bar{y}_{0}<\bar{y}_{0 \text {, crit }}$. For values of $\bar{y}_{0}$ outside this interval, it becomes necessary to keep terms proportional to $\kappa^{2} \epsilon^{2}$ in order to calculate the fastest growing mode, which is no longer the plasmoid instability, but the $\mathrm{KH}$ instability. Retaining the $\kappa^{2} \epsilon^{2}$ terms analytically is difficult; however, we have been able to address the full problem by a direct numerical solution of the linearised equations. Results are shown in section VI. Before discussing those, though, let us present an analytical derivation of the $\mathrm{KH}$ instability of a current sheet valid in the long-wavelength limit $\kappa \epsilon \ll 1$.

\section{KELVIN-HELMHOLTZ INSTABILITY OF THE LAYER}

In this section, we derive an analytical dispersion relation of the Kelvin-Helmholtz $(\mathrm{KH})$ instability of the current sheet valid for perturbations whose wave number is $\kappa \ll \epsilon^{-1}$. This ordering of $\kappa$ is not expected to capture the fastest growing mode, as suggested by the heuristic derivation of section 【C $\left(\kappa_{\max } \sim \epsilon^{-1}\right)$, but we are unable to obtain an analytic solution valid for $\kappa \epsilon \sim 1$. The derivation presented here, however, does reveal a number of interesting features of the $\mathrm{KH}$ instability of the current sheet. The direct numerical solution presented in the next section does of course cover all values of $\kappa$.

We first remind the reader that in a SP reconnection configuration, the outflow velocity profile is maximum at the midplane $(x=0)$ of the sheet and decays to zero away from it. There are, therefore, two shear layers on each side of the sheet, at $x \sim \pm \delta_{\mathrm{CS}}$, where the KH instability may develop — see Fig. 1. These two shear layers will push magnetic fields of opposite sign on each side of $x=0$ towards each other. This will create a current sheet at $x=0$. Thus, resistivity can play an important role in this mode, not at the $\mathrm{KH}$ layers themselves, $x \simeq \pm \delta_{\mathrm{CS}}$, but at $x=0$, where the shear layers interact. This situation is then conceptually similar to the forced reconnection problem treated by Hahm \& Kuslrud 93. (the Taylor problem), where perturbations at some distant boundaries on each side of a rational surface induce reconnection at that surface. Here, the KH instability can be thought of as the equivalent of those perturbations at the boundaries, forcing reconnection at $x=0$.

In [93] (see also 94]) it was found that if the change in the boundaries occurs on a timescale much faster than the resistive one, there will be no reconnection in the early (linear) stage of evolution; the magnetic field will pile up until the current gets sufficiently large for the resistive term to become important. In a somewhat similar fashion, here, whether or not the KH instability induces reconnection depends on how large its growth rate is; for low values of $\kappa \epsilon$, when its growth rate is lower, the pile up of the magnetic field is prevented by reconnection, which can proceed at a rate comparable to the growth rate of the $\mathrm{KH}$ instability. However, for larger values of $\kappa \epsilon$, the $\mathrm{KH}$ instability will be faster than the rate at which reconnection can occur and we expect to find an ideal (i.e., non-reconnecting) mode (though reconnection is expected to start in the nonlinear stage, which we do not address here).

Our formal analysis of this problem again considers three asymptotic regions: the inner region $(|\xi| \ll 1)$, the outer region (or flow shear layer, $|\xi| \sim 1$ ), and the external region $(|\xi| \gg 1)$. Since all the same orderings that led to the simplification of Eqs. (53) 54) in the previous section are still expected to hold here, the equations to solve in each of these regions are the same. In particular, the solution in the the external and outer regions remains unchanged and is given by Eqs. (60) and (71), respectively.

In the inner region, we must solve Eqs. 87 88). There are two cases of interest: when the right-hand side of Eq. (87) is important, and when it is not. The former case occurs at low values of $\kappa \epsilon$, whereupon we simply recover the dispersion relation Eq. (92). This is similar to the resistive-kink-mode solution of Coppi et al. [81]: as $\bar{y}_{0}$ increases and eventually becomes such that $\bar{y}_{0} v_{0}>f_{\infty}$, $\Delta^{\prime}$ [Eq. (78)] transitions from positive to negative via infinity; mathematically, this is equivalent to the well known transition from the very unstable (large- $\Delta^{\prime}$ ) tearing mode to the resistive kink mode [81], except here reconnection is driven by the $\mathrm{KH}$ instability, rather than by the kink mode.

For larger values of $\kappa \epsilon$ (though still requiring that $\kappa \epsilon \ll 1$; we will later determine how large $\kappa$ has to be for the following to hold), we can ignore the right-hand side of Eq. (87) and obtain

$$
\Psi=\frac{f_{0}^{\prime} \xi}{\lambda} \Phi
$$

So, Eq. (88) becomes

$$
\Phi^{\prime \prime}=-\frac{f_{0}^{\prime 2}}{\lambda^{2}} \xi\left(2 \Phi^{\prime}+\xi \Phi^{\prime \prime}\right),
$$

to be solved subject to the boundary condition $\Phi(0)=$ 0 [95]. The solution is:

$$
\Phi^{ \pm}(\xi)=\frac{\Phi_{0}^{\prime} \lambda}{f_{0}^{\prime}} \arctan \left(\frac{f_{0}^{\prime}}{\lambda} \xi\right),
$$

where $\Phi_{0}^{\prime} \equiv \Phi^{\prime}(0)$. The inner layer width is, therefore,

$$
\delta_{\text {inner }}=\frac{\lambda}{f_{0}^{\prime}} .
$$

Using Eq. (101) to substitute for $\Phi$ in Eq. (99), we obtain

$$
\Psi^{ \pm}(\xi)=\Phi_{0}^{\prime} \xi \arctan \left(\frac{f_{0}^{\prime}}{\lambda} \xi\right)
$$


As expected this is a non-reconnecting mode: $\Psi(0)=$ 0 - indeed, this solution is mathematically equivalent to the ideal-kink-mode solution found by Rosenbluth et al. 96]; physically, the difference here is that the drive is the $\mathrm{KH}$ instability.

The solution, Eq. (103), is now matched to the solution in the outer region, Eq. (71). For $\xi \gg 1$, Eq. (103) becomes:

$$
\Psi^{ \pm}(\xi)=\Phi_{0}^{\prime} \xi\left( \pm \frac{\pi}{2}-\frac{\lambda}{f_{0}^{\prime} \xi}\right)
$$

Therefore, we have,

$$
\begin{aligned}
& C_{1}^{ \pm}= \pm \frac{\pi}{2} \frac{\Phi_{0}^{\prime}}{f_{0}^{\prime}}, \\
& C_{2}^{ \pm}=\lambda \Phi_{0}^{\prime} .
\end{aligned}
$$

Substituting these expressions for $C_{1}^{ \pm}, C_{2}^{ \pm}$in Eq. (73), we obtain the final dispersion relation:

$$
\gamma=\lambda \kappa=\frac{\pi}{2} \frac{\bar{y}_{0}^{2} v_{0}^{2}-f_{\infty}^{2}}{f_{0}^{\prime}} \kappa^{2} \epsilon .
$$

This expression shows that an unstable mode exists when $\left|\bar{y}_{0} v_{0}\right|>\left|f_{\infty}\right|$, i.e., above the Alfvén Mach point of the system, $\left|\bar{y}_{0}\right|>\bar{y}_{0 \text {,crit }}$. To determine its region of validity, let us use Eq. (107) and Eq. (102) to compare the the right-hand side of Eq. (87), which we neglect in this derivation, to the first term on the left-hand side of that equation. We then find that this solution is valid for $\epsilon^{-3 / 4} \ll \kappa \ll \epsilon^{-1}$.

Finally, we estimate the value of $\kappa=\kappa_{\text {tr }}$ above which Eq. (107) yields faster growth than the resistive dispersion relation, Eq. (92). This corresponds to a transition from a reconnecting to a non-reconnecting mode. Comparing the two expressions we find

$$
\kappa_{\mathrm{tr}}=\left(\frac{\pi}{2}-\frac{\sqrt{\pi}}{3}\right)^{-3 / 4} \frac{f_{0}^{\prime 5 / 4}}{\left(\bar{y}_{0}^{2} v_{0}^{2}-f_{\infty}^{2}\right)^{3 / 4}} \epsilon^{-3 / 4} .
$$

Wave numbers such that $\kappa>\kappa_{\text {tr }}$ are ideal, nonreconnecting modes (i.e., $\Psi(0)=0$ ). This implies, in particular, that the fastest growing mode, $\kappa_{\max } \sim \epsilon^{-1}$, is an ideal mode, as will be confirmed by the full numerical solution presented in the next section.

\section{NUMERICAL RESULTS}

In this section, we compare the heuristic and the analytical results of sections III IV and $\mathrm{V}$ with the direct numerical solution of the full set of linearized equations:

$$
\begin{aligned}
& \left(\frac{1}{\kappa} \frac{\partial}{\partial \tau}+i \bar{y}_{0} v(\xi)\right) \Psi+\frac{u(\xi)}{\kappa} \Psi^{\prime} \\
& \quad+i \frac{\bar{y}_{0}}{\kappa} g(\xi) \Phi^{\prime}-f(\xi) \Phi=\frac{1}{\kappa}\left(\Psi^{\prime \prime}-\kappa^{2} \epsilon^{2} \Psi\right),
\end{aligned}
$$

$$
\begin{aligned}
& \left(\frac{1}{\kappa} \frac{\partial}{\partial \tau}+i \bar{y}_{0} v(\xi)\right)\left(\Phi^{\prime \prime}-\kappa^{2} \epsilon^{2} \Phi\right) \\
& \quad+\frac{u(\xi)}{\kappa}\left(\Phi^{\prime \prime \prime}-\kappa^{2} \epsilon^{2} \Phi^{\prime}\right)-\frac{u^{\prime \prime}(\xi)}{\kappa} \Phi^{\prime}-i \bar{y}_{0} v^{\prime \prime}(\xi) \Phi= \\
& -f(\xi)\left(\Psi^{\prime \prime}-\kappa^{2} \epsilon^{2} \Psi\right)+i \frac{\bar{y}_{0}}{\kappa} g(\xi)\left(\Psi^{\prime \prime \prime}-\kappa^{2} \epsilon^{2} \Psi^{\prime}\right) \\
& \quad-i \frac{\bar{y}_{0}}{\kappa} g^{\prime \prime}(\xi) \Psi^{\prime}+f^{\prime \prime}(\xi) \Psi,
\end{aligned}
$$

where $\xi=x / \delta_{\mathrm{CS}}, \kappa=k L_{\mathrm{CS}}$ and $\epsilon=\delta_{\mathrm{CS}} / L_{\mathrm{CS}}=S^{-1 / 2}$. These equations follow straightforwardly from Eqs. 447 48) after Fourier decomposing in the $y$-direction: $\delta \psi=$ $\Psi(\xi) \exp (i \kappa y), \quad \delta \phi=-i \Phi(\xi) \exp (i \kappa y)$. The functions $f(\xi), g(\xi), u(\xi)$ and $v(\xi)$ are the normalized SP-like background equilibrium profiles: the reconnecting and reconnected magnetic field components and the inflow and outflow velocity profiles, respectively (see section ЩIA for the normalizations adopted for them). Explicit expressions for these functions, parametrized by the position along the sheet $\bar{y}_{0}=y_{0} / L_{\mathrm{CS}}$, are derived in Appendix $\mathrm{A}$. For the equilibrium adopted, the Alfvén Mach point of the current sheet, defined by $u_{y}\left(\xi=0, \bar{y}_{0, \text { crit }}\right)=$ $B_{y}\left(\xi=\infty, \bar{y}_{0, \text { crit }}\right)$, occurs at $\bar{y}_{0, \text { crit }} \approx 0.61$. Above this point, the upstream magnetic field is no longer able to stabilize the $\mathrm{KH}$ mode.

Eqs. (109 110 are solved in a domain of size $-L_{x} \leq \xi \leq L_{x}$ using a second-order-accurate predictorcorrector numerical scheme. The boundary conditions are $\Psi\left(-L_{x}, t\right)=\Psi\left(L_{x}, t\right)=\Phi\left(-L_{x}, t\right)=\Phi\left(L_{x}, t\right)=0$. The size of the simulation domain $L_{x}$ depends on $\kappa$, with lower values of $\kappa$ requiring larger domains [this is due to the behavior of the eigenfunction in the external region, $\Psi \sim e^{-\kappa \epsilon \xi}$; see Eq. [60)]. Convergence tests were performed to ensure that both the domain size and resolution were appropriate.

\section{A. Plasmoid and $\mathbf{K H}$ instabilities}

We begin by focusing on the inviscid limit, $P m=0$. Plotted in Fig.2(a) are solutions of: (i) the full analytical dispersion relation for the plasmoid instability, Eq. (89), for $\epsilon=10^{-6}$ (i.e., $S=10^{12}$ ) and several different values of $\bar{y}_{0}$ (thin colored lines); (ii) the analytical KH dispersion relation in the resistive limit, Eq. (92) (orange, long-dash-dot line), and in the ideal limit, Eq. (107) (red dotted line), for $\bar{y}_{0}=0.8$ (i.e., beyond the Alfvén Mach point $\left.\bar{y}_{0, \text { crit }}=0.61\right)$. Overplotted (symbols) are the numerical results. The theoretically predicted slopes for the plasmoid instability in the limits $\Lambda \ll 1$ and $\Lambda \rightarrow 1^{-}$ $\left[\kappa^{2 / 3}\right.$ and $\kappa^{-2 / 5}$, respectively; see Eqs. (9192)] are shown by the dotted black lines. The vertical dotted line is at $\kappa \epsilon=1$; all the analytical dispersion relations plotted are only valid for values of $\kappa$ significantly to the left of this line. The vertical dash-dot-dot line shows the position of $\kappa_{\mathrm{tr}}$, the value of $\kappa$ at which a transition from the resistive to the ideal KH mode occurs, as given by Eq. (108).

For $\bar{y}_{0} \leq 0.4$, the agreement between the numerical 

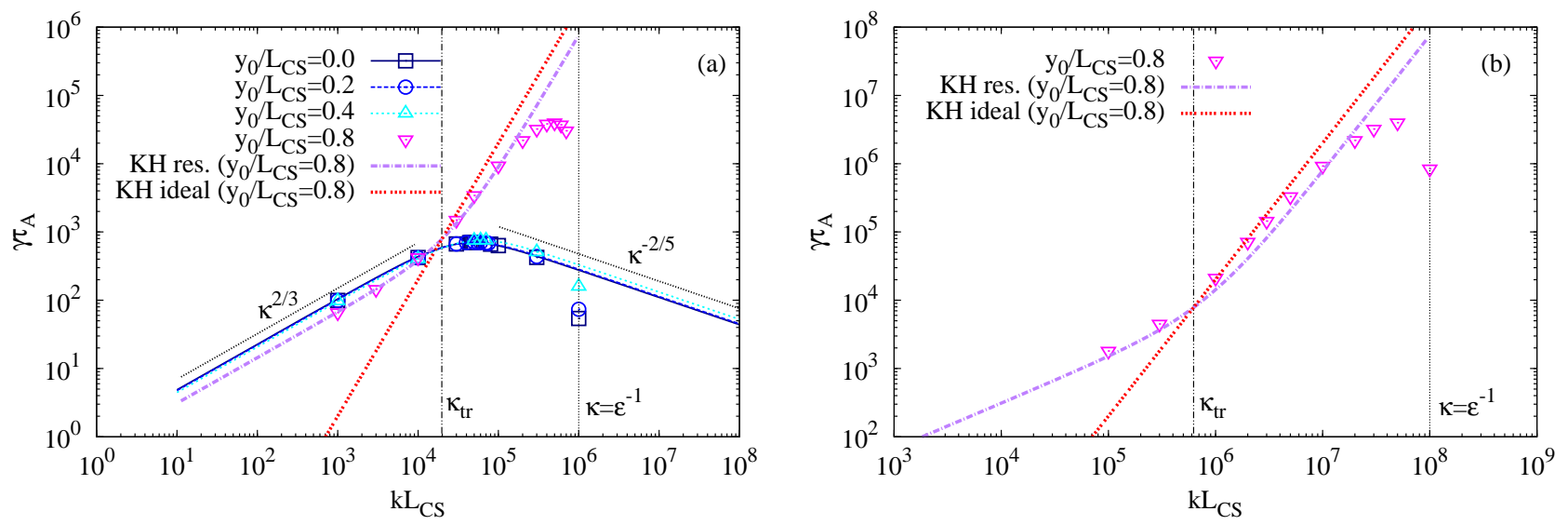

FIG. 2. (a) Growth rate (normalized to the global Alfvén time $\tau_{A}=L_{\mathrm{CS}} / V_{A}$ ) as a function of the wave number $\kappa=k L_{\mathrm{CS}}$ for $\epsilon=10^{-6}$ (i.e., $S=10^{12}$ ) and at positions along the sheet, $\bar{y}_{0}$. Thin colored lines show the solution of the analytical dispersion relation for the plasmoid instability, Eq. (89). The orange long-dash-dot line is the solution of the analytical resisitive KH dispersion relation, Eq. (92), and the red dotted line is the solution of the analytical ideal KH dispersion relation, Eq. (107), both evaluated for $\bar{y}_{0}=0.8$. Symbols are the results of direct numerical integration of the linearized equations. The dotted black lines identify the analytically predicted slopes for the plasmoid instability $\left(\kappa^{2 / 3}\right.$ for $\kappa \lesssim \epsilon^{-3 / 4}$ and $\kappa^{-2 / 5}$ for $\left.\kappa \gg \epsilon^{-3 / 4}\right)$. The vertical dotted line is at $\kappa \epsilon=1$; the vertical dash-dot-dot line identifies $\kappa_{\mathrm{tr}}$ (Eq. (108)). The analytical dispersion relations are numerically solved assuming that the global equilibrium can be approximately described by the analytical solution in the vicinity of the origin derived in Ref. 92]. For this equilibrium, $\bar{y}_{0, \text { crit }}=0.61$. The numerical results use the analytical SP equilibrium calculated in Appendix $\mathrm{A}$ (for which $\bar{y}_{0, \text { crit }}$ is the same). (b) Same as (a) for $\bar{y}_{0}=0.8$ and $\epsilon=10^{-8}$ (i.e., $S=10^{16}$ ).

solution and the analytical plasmoid dispersion relation is very good up to wave numbers approaching $\kappa \epsilon \sim 1$; this is not surprising since we used the ordering $\kappa \epsilon \ll 1$ in our calculation. Importantly, this ordering is indeed respected by $\kappa=\kappa_{\max }$, Eq. (93), which is accurately described by the analytical solution at these values of $\bar{y}_{0}$. Note that the dependence of $\gamma$ on $\bar{y}_{0}$ is very weak, both as predicted by theory [Eq. (94) with $f_{0}^{\prime}, v_{0}$ and $f_{\infty}$ given by Eqs. (A13), (A3) and (A14), respectively] and as determined by the numerical solution (see Fig. (4).

The behavior of the growth rate is distinctly different for $\bar{y}_{0}=0.8$. This value of $\bar{y}_{0}$ is above the Alfvén Mach point, $\bar{y}_{0, \text { crit }}=0.61$, and it is, therefore, in the KH-unstable part of the current sheet. At low values of $\kappa$, the analytical dispersion relation, Eq. (92) (labelled "KH res." in the figure) correctly captures the numerical solution. The transition from the resistive $\mathrm{KH}$ to the ideal $\mathrm{KH}$ mode occurs at $\kappa \approx \kappa_{\text {tr }}$ given by Eq. (108). The ideal KH dispersion relation derived in section $\mathrm{V}$ Eq. (107) (labelled "KH ideal" in the figure), applies for $\kappa>\kappa_{\mathrm{tr}}$, but fails to capture the fastest growing mode, which now occurs when $\kappa \epsilon \sim 1$, as anticipated.

The transition between the resistive and ideal modes is not completely clear in Fig. 2(a); for $\kappa>\kappa_{\text {tr }}$ the numerical data points lie between the ideal and resistive KH lines (red, dotted, and orange, long-dash-dot, respectively) before rolling over and reaching $\gamma_{\max }$ at $\kappa=\kappa_{\max }$. This is because, even at such a large value of $S$, there is not enough scale separation between $\kappa_{\mathrm{tr}} \sim \epsilon^{-3 / 4}$, and $k_{\max } \sim \epsilon^{-1}$ for the ideal solution to match its asymptotic form derived in section $\mathrm{V}$. In order to illustrate clearly this transition, we plot in Fig. 2(b) the dispersion relation obtained at $\bar{y}_{0}=0.8$ for an even smaller (perhaps unrealistically so) $\epsilon=10^{-8}$, i.e., $S=10^{16}$. In this figure, it is clear that the mode becomes ideal for $\kappa>\kappa_{\mathrm{tr}}$, and is correctly described there by the ideal $\mathrm{KH}$ dispersion relation, Eq. (107).

In Fig. 3, we plot the fastest growth rate and the corresponding wave number as functions of the Lundquist number. Whereas the plasmoid instability scalings are obtained at $\bar{y}_{0}=0.0$ (i.e., $\gamma_{\max } \sim S^{1 / 4}$, see Eqs. (9, 94) ), we see that it is the KH scaling that is manifest at $\bar{y}_{0}=0.8$ (i.e., $\gamma_{\max } \sim S^{1 / 2}$, as derived in Eq. (25)).

Plots of $\gamma_{\max }$ and $\kappa_{\max }$ as functions of $\bar{y}_{0}$ at $S=10^{12}$ are shown in Fig. 4. In this figure, the dashed vertical line identifies the position of the Alfvén Mach point, $\bar{y}_{0, \text { crit }} \approx 0.61$. As expected based on the previous discussion, the agreement between the analytical plasmoid dispersion relation, Eq. (89), and the numerical solution is excellent at values of $\bar{y}_{0}<\bar{y}_{0, \text { crit. }}$. As $\bar{y}_{0} \rightarrow \bar{y}_{0, \text { crit }}$, the difference between the analytical and numerical solutions increases and explodes at $\bar{y}_{0}=\bar{y}_{0, \text { crit }}$. The transition to the KH mode happens then; for $\bar{y}_{0} \geq \bar{y}_{0, \text { crit }}$, our simplified analytical theory (section $(\nabla)$ fails to produce a maximum of the growth rate and so cannot be compared to the numerical solution, which confirms that the reason for the failure of the asymptotic theory is that for $\gamma=\gamma_{\max }, \kappa_{\max } \epsilon$ is not small, but approaches values of order unity.

Finally, in Fig. 5, we show the eigenfunctions $\Psi(\xi)$ (left) and $\Phi(\xi)$ (right) corresponding to the fastest growing wavenumber, $\kappa_{\max }$, at $S=10^{12}$. These plots are con- 

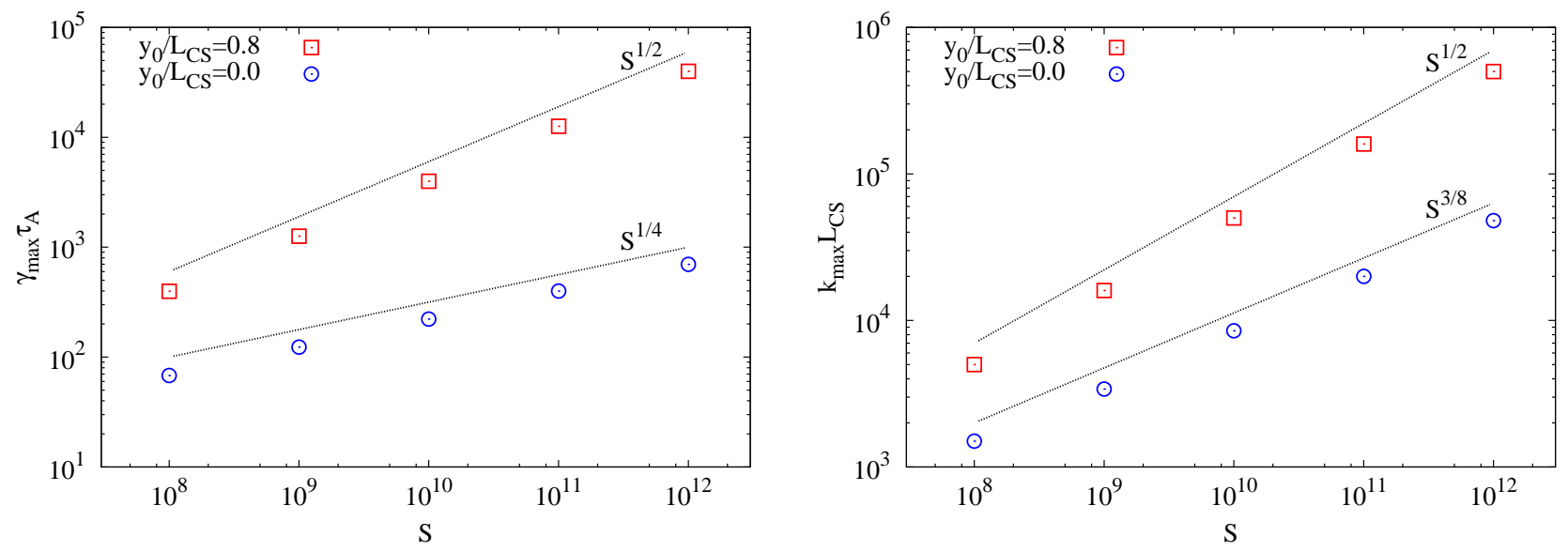

FIG. 3. Maximum growth rate (left) and the corresponding wave-number (right) as functions of the Lundquist number $S$, for $\bar{y}_{0}=0.0$ and $\bar{y}_{0}=0.8$. The plasmoid instability $\left[\gamma_{\max } \sim S^{1 / 4}, \kappa_{\max } \sim S^{3 / 8}\right.$; see Eqs. (9), 940] observed at $\bar{y}_{0}=0.0$ is superseded by the KH instability $\left[\gamma_{\max } \sim S^{1 / 2}, \kappa_{\max } \sim S^{1 / 2}\right.$; see Eq. (25)] at $\bar{y}_{0}=0.8$.
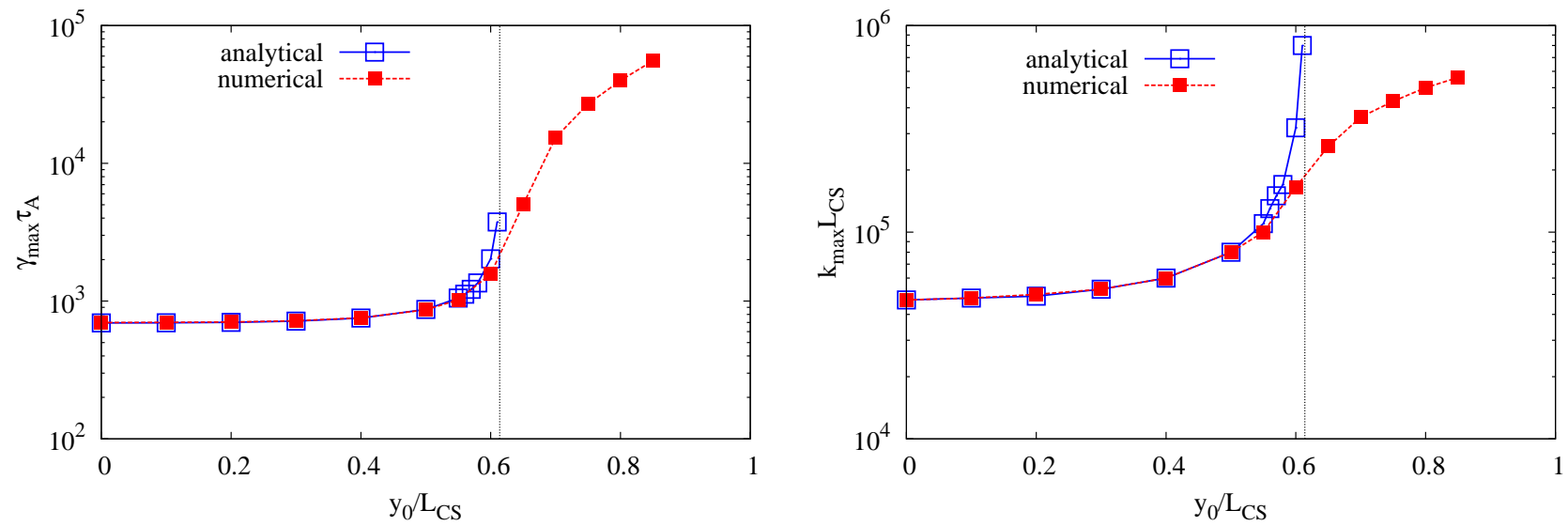

FIG. 4. Maximum growth rate (left) and the corresponding wave-number (right) as functions of the position $\bar{y}_{0}$ along the sheet, for $\epsilon=10^{-6}$ (i.e., $S=10^{12}$ ). The analytic solution is given by Eqs. (93 94). The vertical dotted line identifies the position of $\bar{y}_{0, \text { crit }}$ for the equilibrium parameters specified in Eq. A14.

structed from runs at the values of $\bar{y}_{0}$ plotted in Fig. [4 We see that the eigenfunctions undergo an abrupt change at $\bar{y}_{0} \sim 0.6$, where the Alfvén Mach point of the system is located (identified by the dashed white line). Close inspection of the $\Psi$ eigenfunctions reveals that for $\bar{y}_{0}>0.6$, $\Psi(0) \rightarrow 0$, as predicted in section $\mathrm{V}$ i.e., the most unstable mode is ideal. Visible in the plot of the $\Phi$ eigenfunction beyond the Alfvén Mach point is the formation of structure at each of the KH-unstable shear layers, located at $x / \delta_{\mathrm{CS}} \approx \pm 1$. For a clearer observation of both these properties of the eigenfunctions, we plot in Fig. 6 one-dimensional cuts of Fig. 5 , taken at $\bar{y}_{0}=0.4$ (i.e., below the Alfvén Mach point) and $\bar{y}_{0}=0.8$ (i.e., above the Alfvén Mach point). As seen, for $\bar{y}_{0}=0.4, \Psi$ is finite at $\xi=0$, whereas it is zero at $\bar{y}_{0}=0.8$, in agreement with the analytical theory of section $\mathrm{V}$ and the prediction that above $\bar{y}_{0, \text { crit }}$ the fastest growing mode is non-reconnecting. Furthermore, the broadening of the $\Psi$ eigenfunction around $\xi=0$ at $\bar{y}_{0}=0.8$ suggests the pileup of the magnetic field that we discussed in section $\nabla$

\section{B. Effect of viscosity}

In order to address the effect of viscosity on the plasmoid instability, the term $P m\left(\Phi^{\prime \prime \prime \prime}-2 \kappa^{2} \epsilon^{2} \Phi^{\prime \prime}+\kappa^{4} \epsilon^{4} \Phi\right) / \kappa$, where $P m=\nu / \eta$, is added to the right-hand side of Eq. (110), and two additional boundary conditions are used: $\Phi^{\prime \prime}\left(-L_{x}, t\right)=\Phi^{\prime \prime}\left(L_{x}, t\right)=0$. We follow the generalization of the SP scalings to plasmas where $P m \gg 1$ derived in Ref. [36]: namely, we must scale the electric field at the origin as $E_{0} / \mathrm{Pm}^{1 / 4}$, and the width of the current layer as $\delta_{\mathrm{CS}} \rightarrow \delta_{S P} P m^{1 / 4}$, where $\delta_{S P} / L_{\mathrm{CS}}=S^{-1 / 2}$. There- 

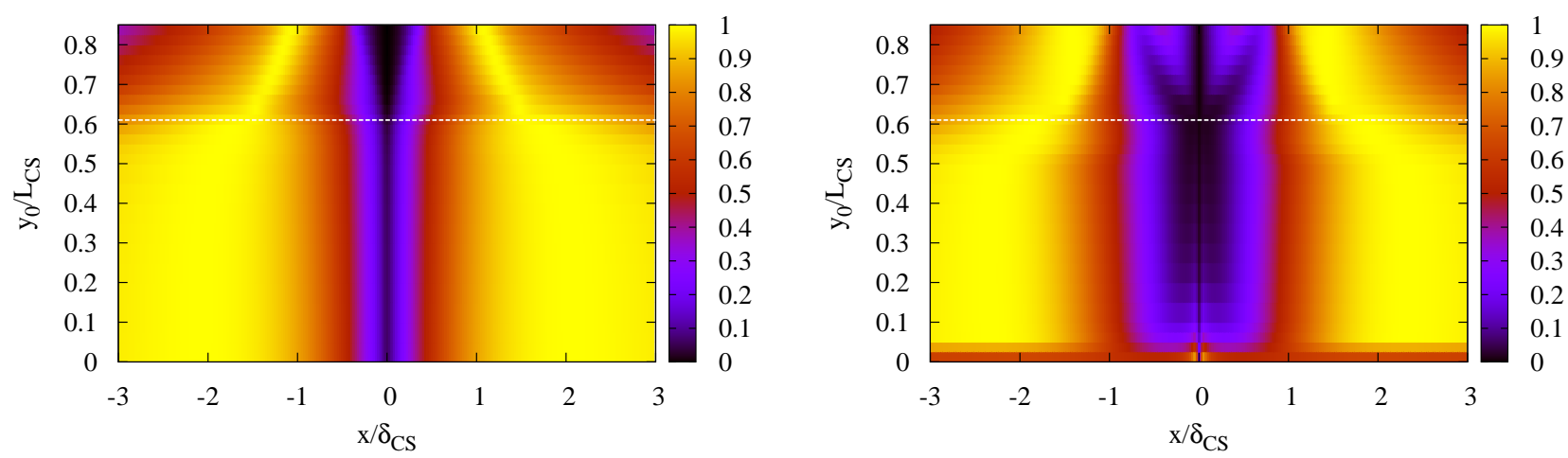

FIG. 5. Eigenfunctions $\left(|\Psi|\right.$, left, and $|\Phi|$, right, normalized to their respective maxima) for $\kappa=\kappa_{\max }$ at $\epsilon=10^{-6}$ (i.e., $\left.S=10^{12}\right)$. These plots are constructed from runs at the values of $\bar{y}_{0}$ shown in Fig. 4 In all runs, the size of the simulation domain is $L_{x}=100$, and the resolution is $\Delta x=0.0125$. Only a fraction of the simulation domain is shown. The horizontal dashed white line shows the location of the Alfvén Mach point, $\bar{y}_{0}=\bar{y}_{0, \text { crit }}$.
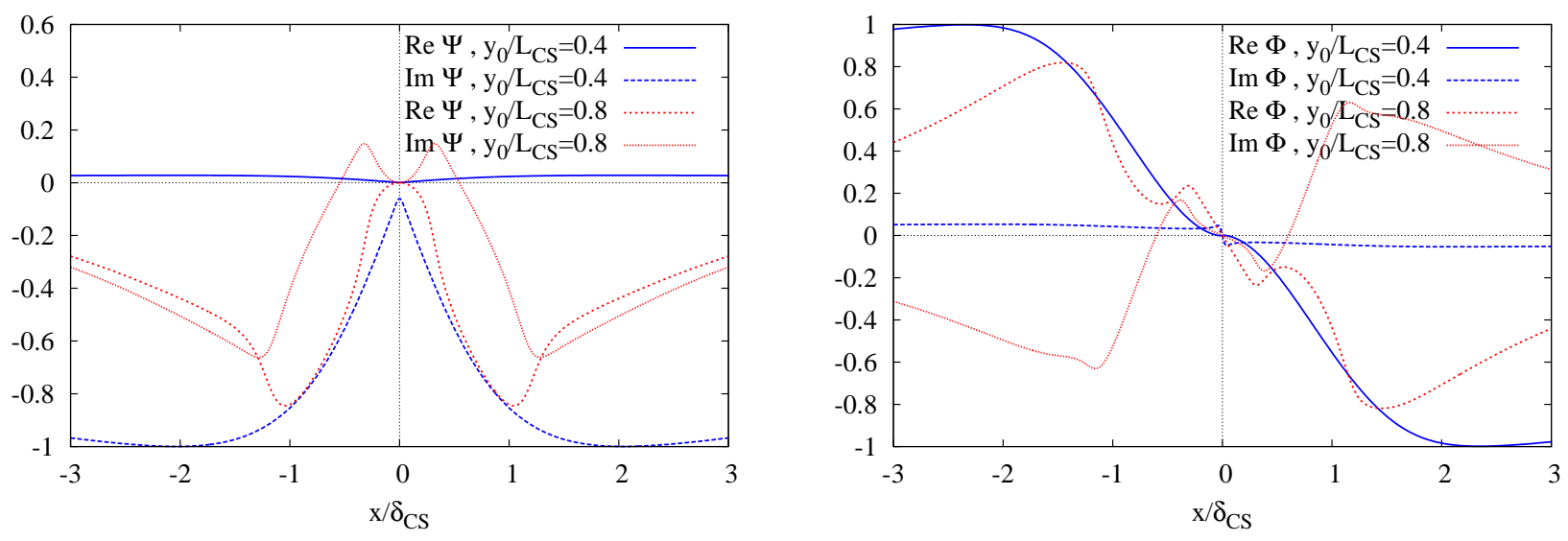

FIG. 6. Real and imaginary parts of the eigenfunctions for $\kappa=\kappa_{\max }$ at $\epsilon=10^{-6}$ (i.e., $S=10^{12}$ ) and $\bar{y}_{0}=0.4$ and $\bar{y}_{0}=0.8$. Only a fraction of the simulation domain is shown.

fore, we rescale the parameter $\epsilon=\delta_{\mathrm{CS}} / L_{\mathrm{CS}}$ according to $\epsilon \rightarrow \epsilon_{S P} P m^{1 / 4}$, where $\epsilon_{S P}=\delta_{S P} / L_{\mathrm{CS}}=S^{-1 / 2}$.

Plotted in Fig. 7 are the maximum growth rate and the corresponding wave-number as a function of $\mathrm{Pm}$, for $S=10^{12}$ and $\bar{y}_{0}=0$. Both $\kappa_{\max }$ and $\gamma_{\max }$ are seen to decrease with increasing Prandtl number; a good fit to the data is given by $\gamma_{\max } \propto P m^{-5 / 8}$ and $\kappa_{\max } \propto P m^{-3 / 16}$. The scaling of $\gamma_{\max }$ and $\kappa_{\max }$ with the Lundquist number at $P m=30$ is shown in Fig. 8 . We see that the $S$ dependence of the maximum growth rate and of the corresponding wavenumber remains unchanged at large $P m$, i.e., $\gamma_{\max } \propto S^{1 / 4}$ and $\kappa_{\max } \propto S^{3 / 8}$. These results agree exactly with the power laws derived in section II] Eqs. (18 19).

\section{SUMMARY}

In this paper, a two-dimensional linear theory of the instability of large-aspect-ratio, Sweet-Parker-like current sheets is presented. This work is a direct generalization of our previous results [6] (Paper I), where the simple equilibrium used was only a good model of a current sheet in the immediate vicinity of $y=0$ ( $y$ is the outflow direction).

In the work presented here a general 2D SP-like current sheet equilibrium is considered. As in Paper I, we conclude that large-aspect-ratio Sweet-Parker current sheets are violently unstable to high-wave-number tearing-like perturbations, and the same scalings of the growth rate with the Lundquist number $S=L V_{A} / \eta$ are obtained here: $\gamma_{\max } \tau_{A} \sim S^{1 / 4}$ and $k_{\max } L_{\mathrm{CS}} \sim S^{3 / 8}-$ see Eqs. 93 94). The plasmoid chain is formed inside a 

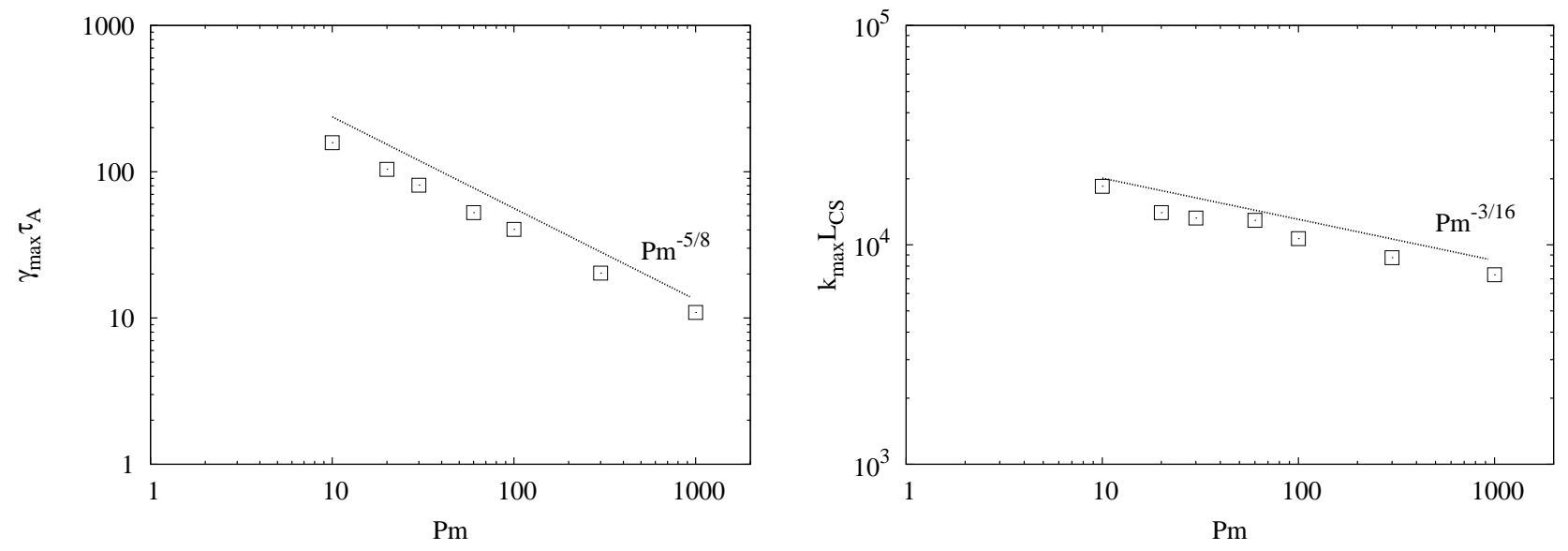

FIG. 7. Maximum growth rate (left) and the corresponding wave-number (right) as a function of the Prandtl number Pm for $\epsilon=10^{-6}$ (i.e., $S=10^{12}$ ) and $\bar{y}_{0}=0$.
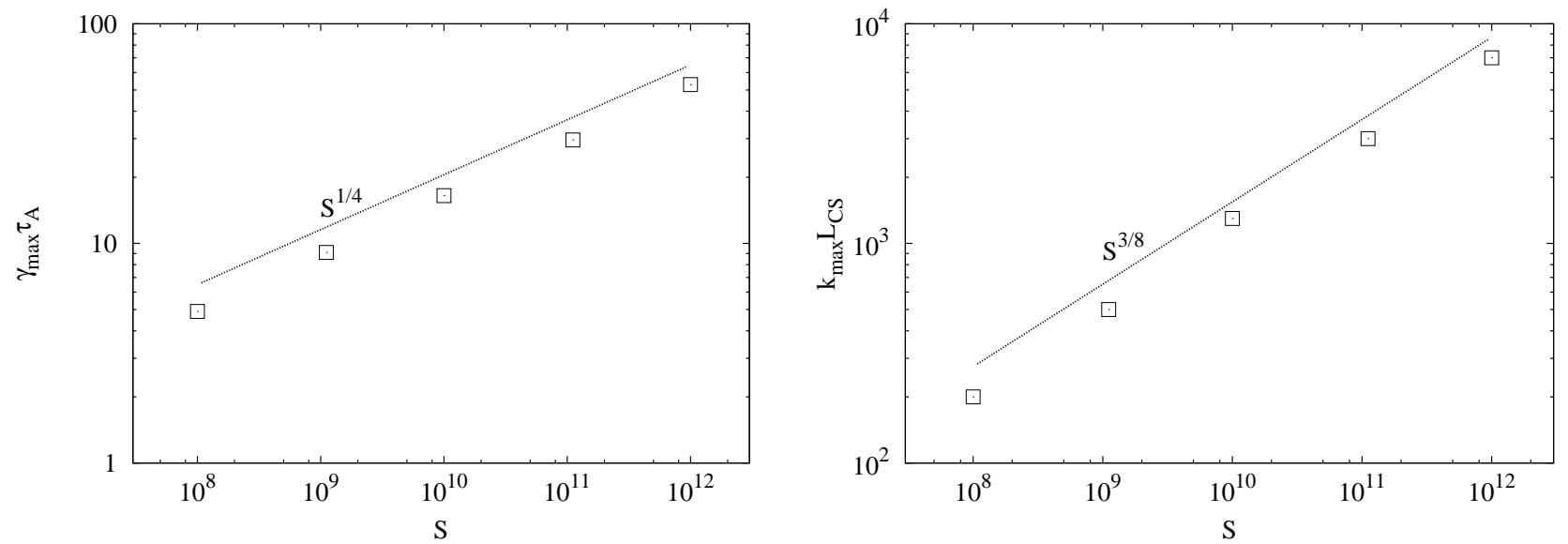

FIG. 8. Maximum growth rate (left) and the corresponding wave-number (right) as a function of the Lundquist number $S$ at $P m=30$ and $\bar{y}_{0}=0$.

boundary layer whose width scales as $\delta_{\text {inner }} / \delta_{\mathrm{CS}} \sim S^{-1 / 8}$. These scalings have been confirmed via direct numerical simulation [43, 77].

The more general approach employed in this paper has allowed us to calculate the growth rate of the plasmoid instability as a function of the position along the current sheet, $y_{0}$. The dependence of $\gamma_{\max }$ and $k_{\max }$ on $y_{0}$ is a nontrivial function of the particular equilibrium considered and, in the absence of a known analytical solution to the SP problem, cannot be evaluated explicitly. However, for $y_{0} / L_{\mathrm{CS}} \ll 1$ we make use of the semi-analytical results of Uzdensky and Kulsrud 92] and present an exact solution - Eqs. (97 98). The most unstable wavenumber and corresponding growth rate are then found to increase with distance from the center. Under general conditions (Syrovatskii-like upstream magnetic-field profile and outflow profile increasing monotonically along the layer), we show that the same result holds true at arbitrary $y_{0} / L_{\mathrm{CS}} \sim 1$. This finding is somewhat counter- intuitive: a priori, one could expect that the increasing strength of the reconnected field along the sheet, as well as the shear in the ouflow (in the $y$ direction), would provide a stabilising effect. Our calculation shows, however, that both are irrelevant to the instability. An intuitive understanding of why that should be so can be gained by comparing the strength of the upstream and the downstream magnetic fields at the boundary of the inner (plasmoid) layer, $\xi=\delta_{\text {inner }}$ :

$$
\left.\frac{B_{y}}{B_{x}}\right|_{x \sim \delta_{\text {inner }}} \sim \frac{\delta_{\text {inner }}}{\delta_{\mathrm{CS}}} S^{1 / 2} \sim S^{3 / 8} \gg 1,
$$

i.e., even at the scale of the inner layer the reconnected field $B_{x}$ is completely overwhelmed by the reconnecting field $B_{y}$. The gradient of the background outflow in the $y$ direction, whose length scale is $\sim L_{\mathrm{CS}}$, is also unimportant because $k_{\max } L_{\mathrm{CS}} \gg 1$ everywhere in the sheet. At the periphery of the sheet, for $y_{0}>y_{0, \text { crit }}$, where $y_{0, \text { crit }} / L_{\mathrm{CS}}$ is equilibrium-dependent but otherwise 
$\mathcal{O}(1)$, the current sheet becomes unstable to the KelvinHelmholtz (KH) instability driven by the velocity shear between the Alfvénic reconnection outflow and the stationary upstream plasma. This occurs because the magnitude of the upstream magnetic field is a decreasing function of the outflow coordinate $y$ and eventually becomes smaller than the outflow speed (which is an increasing function of $\left.y / L_{\mathrm{CS}}\right)$. At, and beyond, the Alfvén Mach point, where this happens, the magnetic field can no longer stabilize the current sheet against the KH instability.

We find that the KH instability of the sheet can either be resistive (i.e., induce reconnection at $x=0$ ), or ideal (no reconnection), with lower values of $k L_{\mathrm{CS}}$ corresponding to the former, and larger values to the latter. The fastest growing $\mathrm{KH}$ mode, $k_{\max } L_{\mathrm{CS}} \sim S^{1 / 2}$ (i.e., $k_{\max } \delta_{\mathrm{CS}} \sim 1$ ), is an ideal, non-reconnecting mode. This is because reconnection cannot occur at the fast rates required by the fastest growing $\mathrm{KH}$ mode. A useful analogy can be made with the Taylor (forced reconnection) problem [93]: since there are two shear layers, one on each side of the current sheet, the KH instability of the sheet is conceptually similar to a situation where perturbations at distant walls attempt to drive reconnection at a rational surface. In the Taylor problem, it is also found that the perturbations at the walls do not drive reconnection in the initial stage. However, the same analogy suggests that as the ideal KH mode evolves into the nonlinear regime, it will cause the upstream magnetic field to pile-up in the current layer, eventually leading to its reconnection. This KH-driven reconnection that occurs at $y_{0}>y_{0, \text { crit }}$ will give rise to a plasmoid chain, just as the "pure" plasmoid instability that is found at $y_{0}<y_{0, \text { crit }}$. Therefore, in practice, it may be difficult to distinguish between the two situations.

It is also worth noting that the basic $\mathrm{KH}$ instability mechanism that we have described here is completely general, i.e., it should apply to any reconnecting current sheet, not just to those that can be described by the reduced MHD framework that we have adopted here: the only ingredient it requires is the existence of an Alfvénic Mach point somewhere along the layer. This should be a generic feature of most reconnecting current sheets. Whether the layer is collisional or collisionless may affect the dynamics of the $\mathrm{KH}$ instability, but its existence is not dependent on the plasma collisionality. In this respect, our findings may be related to recent observations of the $\mathrm{KH}$ instability in collisionless simulations of guide-field reconnection [97].

Finally, the effect of viscosity on the plasmoid instability has been addressed via numerical integration of the linearised set of equations. Our results are that in the limit $P m=\nu / \eta \gg 1$, the fastest growth rate and wavenumber of the plasmoid instability scale as:

$$
\begin{gathered}
\gamma_{\max } \sim S^{1 / 4} \mathrm{Pm}^{-5 / 8} \sim L_{\mathrm{CS}}^{1 / 4} V_{A}^{1 / 4} \eta^{3 / 8} \nu^{-5 / 8}, \\
\kappa_{\max } \sim S^{3 / 8} \mathrm{Pm}^{-3 / 16} \sim L_{\mathrm{CS}}^{3 / 8} V_{A}^{3 / 8} \eta^{-3 / 16} \nu^{-3 / 16} .
\end{gathered}
$$

We have not performed a rigorous analytical calculation of the plasmoid instability in this limit, but we have been able to recover these scalings in a non-rigorous way from known results on the visco-tearing and resistivekink modes 84], via the rescaling of the background magnetic shear length $a \rightarrow \delta_{\mathrm{CS}} \sim L_{\mathrm{CS}} S^{-1 / 2} \mathrm{Pm}^{1 / 4}$ [36]. Although these scalings are only expected to apply for $S \gg S_{\text {crit }}, P m \gg 1$, where $S_{\text {crit }}$ is the critical value of the Lundquist number for the current sheet to be plasmoidunstable, they lead to the prediction that

$$
S_{\text {crit }} \sim 10^{4} \mathrm{Pm}^{1 / 2}, \quad \mathrm{Pm} \gg 1 .
$$

This result, as well as those of Eqs. (112 113) are concrete predictions that can be tested in direct numerical simulations of MHD reconnection in the large magnetic Prandtl number regime.

\section{ACKNOWLEDGMENTS}

The authors would like to thank S. C. Cowley, R. Samtaney and J. B. Taylor for important discussions. The work of N.F.L. was supported by Fundação para a Ciência e a Tecnologia (Ciência 2008 and Grant no. PTDC/FIS/118187/2010) and by the European Communities under the contract of Association between EURATOM and IST. The views and opinions expressed herein do not necessarily reflect those of the European Comission. A.A.S. was supported in part by an STFC Advanced Fellowship and the STFC Grant ST/F002505/2. D.A.U. was supported by NSF Grant PHY-0903851. N.F.L. and D.A.U. thank the Leverhulme Trust International Network for Magnetized Plasma Turbulence for travel support. Numerical work was carried out at the IST cluster (Instituto Superior Técnico), Newhydra (University of Oxford) and Verus (University of Colorado).

\section{Appendix A: Equilibrium Considerations}

Exact two-dimensional solutions of Eqs. (27 28) describing a Sweet-Parker-like reconnecting current sheet are not known. In principle, these can be obtained by substituting the expressions for $\psi$ and $\phi$ given by Eqs. (30 31) into Eqs. (27 28) and equating equal powers of $\left(y-y_{0}\right) / L_{\mathrm{CS}}=\bar{y}-\bar{y}_{0}$. To lowest order in $\bar{y}-\bar{y}_{0}$, we obtain the following equations:

$$
\begin{aligned}
u(\xi) f(\xi)-\bar{y}_{0}^{2} v(\xi) g(\xi)= & f^{\prime}(\xi)-\bar{E}_{0}, \\
u(\xi) v^{\prime \prime}(\xi)-v(\xi) u^{\prime \prime}(\xi)= & g(\xi) f^{\prime \prime}(\xi)-f(\xi) g^{\prime \prime}(\xi)+ \\
& \operatorname{Pm} v^{\prime \prime \prime}(\xi),
\end{aligned}
$$

where we have used the normalizations of Eq. (33), neglected terms of order $\epsilon^{2}$, and defined the normalized electric field $\bar{E}_{0}=L_{\mathrm{CS}} E_{0} /\left(B_{0}^{2} \delta_{\mathrm{CS}}\right)$.

Evaluated at $\xi=0$, Eq. (A1) yields:

$$
f_{0}^{\prime}=\bar{E}_{0}-\bar{y}_{0}^{2} v_{0} g_{0},
$$


whereas for $\xi \gg 1$ we obtain from the same equation

$$
u_{\infty}=\frac{\bar{E}_{0}}{f_{\infty}}
$$

These expressions are exact; however, we see that Eqs. A1 A2 are not a closed set, since there are only two equations and four unknowns: $f(\xi), g(\xi)$ (the normalized reconnecting and reconnected magnetic field profiles, respectively), and $u(\xi), v(\xi)$ (the normalized inflow and outflow velocity profiles, respectively). This closure problem is introduced by the expansion in $\left(\bar{y}-\bar{y}_{0}\right)$ (recall the discussion of section (II). In order to obtain a SP-like equilibrium, which we require for our numerical solution, one has to close Eqs. A1 A2 , e.g., by guessing two of the four unknown functions, and solving those equations for the other two. Any model of the equilibrium that can be found in this way is necessarily non-unique (i.e., dependent on the guesses required for the closure); however, we will see in what follows that a qualitatively satisfactory model of a SP current sheet can be obtained by this procedure.

Let us introduce an auxiliary function, $s(\xi)$, defined by the following equation:

$$
g(\xi)=\frac{u_{\infty}}{f_{\infty}} v(\xi)-s(\xi) .
$$

Then, from Eq. A1 we obtain

$$
\begin{aligned}
v(\xi)= & \frac{f_{\infty}}{u_{\infty}} \frac{s(\xi)}{2} \\
& \pm \sqrt{\frac{f_{\infty}^{2}}{u_{\infty}^{2}} \frac{s^{2}(\xi)}{4}+\frac{f_{\infty}}{u_{\infty}} \frac{u(\xi) f(\xi)-f^{\prime}(\xi)+\bar{E}_{0}}{\bar{y}_{0}^{2}}}
\end{aligned}
$$

Eq. A2 can also be easily solved in the limit $P m=0$ (viscous effects in the equilibrium that we are about to derive can be modelled by a rescaling of the current sheet thickness, the outflow speed and the reconnection electric field according to the SP relationships in the viscous regime derived in [36]). Using Eq. (A5), Eq. (A2 becomes

$$
s^{\prime \prime}(\xi)=s(\xi) \frac{f^{\prime \prime}(\xi)}{f(\xi)},
$$

to be solved subject to the boundary conditions $s(0)=$ $u_{\infty} v_{0} / f_{\infty}-g_{0}$, where $v_{0}=v(0)$ and $g_{0}=g(0)$, and $s^{\prime}(0)=0$ (we demand that both $v(\xi)$ and $g(\xi)$ are even functions).

The general solution to this equation is

$$
s(\xi)=C_{1} f(\xi)+C_{2} f(\xi) \int^{\xi} \frac{d \xi^{\prime}}{f^{2}\left(\xi^{\prime}\right)} .
$$

[The lower limit of integration on the last term on the right-hand side of this expression need not be specified as $C_{1}$ can be redefined to absorb the difference between different lower limits; note however that we take the lower limit to be finite, i.e., neither 0 nor $\infty$.]

At this stage, the equilibrium problem is solved if we provide functional forms for the reconnecting magnetic field, $f(\xi)$, and for the inflow velocity profile, $u(\xi)$. The simplest choice for $f(\xi)$ is the "Harris sheet" [98]:

$$
f(\xi)=f_{\infty} \tanh \left(\frac{f_{0}^{\prime}}{f_{\infty}} \xi\right) .
$$

A qualitatively plausible choice for $u(\xi)$ is

$$
u(\xi)=-u_{\infty} \frac{f(\xi)}{f_{\infty}}
$$

Substituting Eq. A9 into Eq. (A8) and evaluating the integral explicitly, we obtain:

$$
s(\xi)=\left(g_{0}-\frac{u_{\infty}}{f_{\infty}} v_{0}\right)\left[\frac{f_{0}^{\prime}}{f_{\infty}} \xi \tanh \left(\frac{f_{0}^{\prime}}{f_{\infty}} \xi\right)-1\right],
$$

where the constants of integration $C_{1}, C_{2}$ have been chosen to satisfy the boundary conditions we specified for $s(\xi)$. Substituting Eqs. A9 A11 into Eqs. A5 A6) yields explicit expressions for the two remaining unknowns, the reconnected magnetic field $g(\xi)$ and the outflow velocity profile, $v(\xi)$. Although it is not particularly enlightening to write down these expressions in explicit form, it is useful to evaluate $g(\xi)$ for $\xi \gg 1$. It is

$$
\left.g(\xi)\right|_{\xi \gg 1} \approx \pm\left(\frac{u_{\infty}}{f_{\infty}} v_{0}-g_{0}\right) \frac{f_{0}^{\prime}}{f_{\infty}} \xi \equiv \pm g_{\infty}^{\prime} \xi .
$$

This expression is used in section IVA to estimate the magnitude of $g(\xi)$ for $\xi \gg 1$.

The last step in obtaining an analytical SP-like equilibrium solution consists of determining $E_{0}, v_{0}, g_{0}$ and $f_{\infty}$, all of which can in principle be functions of $\bar{y}_{0}$. A reasonable choice for $f_{\infty}$ is a Syrovatskii-like profile [86]:

$$
f_{\infty}=\sqrt{1-\bar{y}_{0}^{2}}
$$

As for $E_{0}, v_{0}, g_{0}$, their values at $\bar{y}_{0}=0$ have been calculated semi-analytically in Ref. 92] [99]:

$$
\begin{aligned}
\bar{E}_{0}\left(\bar{y}_{0}=0\right) & =1.075 ; \\
g_{0}\left(\bar{y}_{0}=0\right) & =0.642 ; \\
v_{0}\left(\bar{y}_{0}=0\right) & =1.286 .
\end{aligned}
$$

The simplest choice is to assume that these values are constant along the sheet [note, though, that a linearly increasing dependence of the outflow and reconnected field profiles is already included in the normalizations, Eq. (33)].

Examples of the equilibrium profiles obtained in this fashion are shown in Fig. 9, for $\bar{y}_{0}=0.4$ (left) and $\bar{y}_{0}=0.8$ (right) (in Eq. (A6), the solution with the ' + ' sign is chosen). We see that these profiles retain all the qualitative features expected of a true SP equilibrium. 

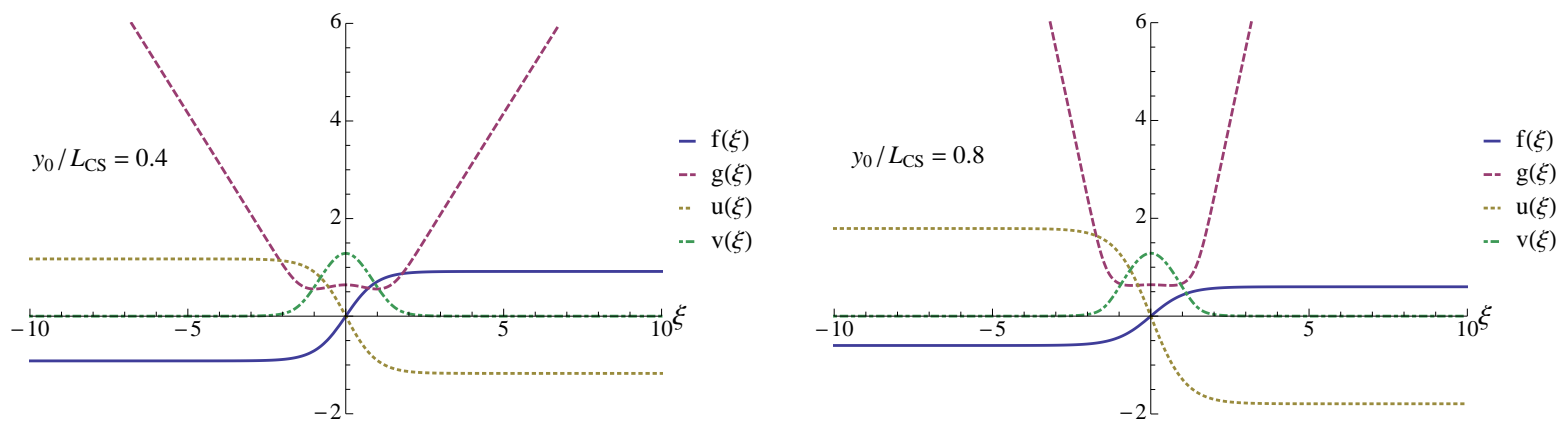

FIG. 9. Analytic SP-like equilibrium profiles (Eqs. A5), A6), A9), A10) evaluated for $\bar{y}_{0}=0.4$ (left), and $\bar{y}_{0}=0.8$ (right). These equilibria are obtained by chosing the functional form of the upstream magnetic field, $f(\xi)$, and imposing that the inflow profile be such that $u(\xi)=-u_{\infty} f(\xi) / f_{\infty}$. The lowest order (in $\left(y-y_{0}\right) / L_{\mathrm{CS}}$ ) Ohm's law and momentum equation can then be solved for the two remaining unknowns, namely the reconnected magnetic field, $g(\xi)$, and the outflow, $v(\xi)$.

Note that for these parameters, the Alfvén Mach point of the system occurs at $\bar{y}_{0, \text { crit }}=0.61$.

The solution found here can be viewed as a generalization to the entire current sheet of the equilibrium derived by Biskamp [88], which is only applicable for $\bar{y}_{0}=0$. As mentioned above, the equilibrium profiles obtained by this procedure, though exact, are not unique, since they depend on the guesses for $f(\xi), u(\xi)$; another ansatz can, in principle, yield a different, but equally plausible, equi- librium. For the purposes of this paper, however, we do not believe this to be a serious constraint since we expect both the plasmoid and the KH instabilities to be largely independent of the fine details of the background profiles; this certainly seems to be true for the plasmoid instability, as is suggested by the agreement between the theoretical predictions of Paper I using a very simplified equilibrium and subsequent numerical studies [43, 47]. The profiles we have derived are a convenient model for solving the linear problem, as we do in section VI.
[1] D. Biskamp, Magnetic Reconnection in Plasmas (Cambridge University Press, 2000).

[2] E. Priest and T. Forbes, Magnetic Reconnection (Cambridge University Press, 2000).

[3] E. G. Zweibel and M. Yamada, Annu. Rev. Astron. Astrophys. 47, 291 (2009)

[4] M. Yamada, R. Kulsrud, and H. Ji, Rev. Mod. Phys. 82, 603 (2010)

[5] K. Shibata and T. Magara, Liv. Rev. Solar Phys. 8, 6 (2011)

[6] M. Oieroset, T. D. Phan, M. Fujimoto, R. P. Lin, and R. P. Lepping, Nature 412, 414 (2001).

[7] R. J. Hastie, Astrophys. Space Sci. 256, 177 (1997)

[8] H. P. Furth, J. Killeen, and M. N. Rosenbluth, Phys. Fluids 6, 459 (1963).

[9] P. H. Rutherford, Phys. Fluids 16, 1903 (1973)

[10] P. M. Nilson, L. Willingale, M. C. Kaluza, C. Kamperidis, S. Minardi, M. S. Wei, P. Fernandes, M. Notley, S. Bandyopadhyay, M. Sherlock, R. J. Kingham, M. Tatarakis, Z. Najmudin, W. Rozmus, R. G. Evans, M. G. Haines, A. E. Dangor, and K. Krushelnick, Phys. Rev. Lett. 97, 255001 (2006)

[11] L. Willingale, P. M. Nilson, M. C. Kaluza, A. E. Dangor, R. G. Evans, P. Fernandes, M. G. Haines, C. Kamperidis, R. J. Kingham, C. P. Ridgers, M. Sherlock, A. G. R. Thomas, M. S. Wei, Z. Najmudin, K. Krushelnick, S. Bandyopadhyay, M. Notley, S. Minardi, M. Tatarakis, and W. Rozmus, Phys. Plasmas 17, 043104 (2010)
[12] Q.-L. Dong, S.-J. Wang, Q.-M. Lu, C. Huang, D.-W. Yuan, X. Liu, X.-X. Lin, Y.-T. Li, H.-G. Wei, J.-Y. Zhong, J.-R. Shi, S.-E. Jiang, Y.-K. Ding, B.-B. Jiang, K. Du, X.-T. He, M. Y. Yu, C. S. Liu, S. Wang, Y.-J. Tang, J.-Q. Zhu, G. Zhao, Z.-M. Sheng, and J. Zhang, Phys. Rev. Lett. 108, 215001 (2012).

[13] J. Lin, S. R. Cranmer, and C. J. Farrugia, J. Geophys. Res. 113, 11107 (2008)

[14] Q. Zong, T. A. Fritz, Z. Y. Pu, S. Y. Fu, D. N. Baker, H. Zhang, A. T. Lui, I. Vogiatzis, K. Glassmeier, A. Korth, P. W. Daly, A. Balogh, and H. Reme, Geophys. Res. Lett. 31, 18803 (2004).

[15] J. P. Eastwood, D. G. Sibeck, J. A. Slavin, M. L. Goldstein, B. Lavraud, M. Sitnov, S. Imber, A. Balogh, E. A. Lucek, and I. Dandouras, Geophys. Res. Lett. 32, 11105 (2005).

[16] L. Chen, A. Bhattacharjee, P. A. Puhl-Quinn, H. Yang, N. Bessho, S. Imada, S. Muhlbachler, P. W. Daly, B. Lefebvre, Y. Khotyaintsev, A. Vaivads, A. Fazakerley, and E. Georgescu, Nat. Phys. 4, 19 (2008).

[17] J. Lin, Y. Ko, L. Sui, J. C. Raymond, G. A. Stenborg, Y. Jiang, S. Zhao, and S. Mancuso, Astrophys. J. 622, 1251 (2005).

[18] A. Ciaravella and J. C. Raymond, Astrophys. J. 686, 1372 (2008).

[19] A. Bemporad, Astrophys. J. 689, 572 (2008)

[20] J. Lin, J. Li, Y. Ko, and J. C. Raymond, Astrophys. J. 693, 1666 (2009).

[21] N. Nishizuka, H. Takasaki, A. Asai, and K. Shibata, 
Astrophys. J. 711, 1062 (2010)

[22] S. Takasao, A. Asai, H. Isobe, and K. Shibata, Astrophys. J. 745, L6 (2012).

[23] A. J. Donné, J. C. van Gorkom, V. S. Udintsev, C. W. Domier, A. Krämer-Flecken, N. C. Luhmann, and F. C. Schüller, Phys. Rev. Lett. 94, 85001 (2005)

[24] Y. Liang, H. Koslowski, A. Krämer-Flecken, O. Zimmermann, K. Löwenbrück, G. Bertschinger, and R. Wolf, Nucl. Fusion 47, L21 (2007)

[25] F. Salzedas and Jet EFDA Contributors, Phys. Plasmas 18, 0701 (2011).

[26] H. K. Park, N. C. Luhmann, A. J. H. Donné, I. G. J. Classen, C. W. Domier, E. Mazzucato, T. Munsat, M. J. van de Pol, and Z. Xia, Phys. Rev. Lett. 96, 195003 (2006)

[27] H. K. Park, A. J. H. Donné, N. C. Luhmann, I. G. J. Classen, C. W. Domier, E. Mazzucato, T. Munsat, M. J. van de Pol, and Z. Xia, Phys. Rev. Lett. 96, 195004 (2006).

[28] T. Munsat, H. K. Park, I. G. J. Classen, C. W. Domier, A. J. H. Donné, N. C. Luhmann, E. Mazzucato, M. J. van de Pol, and T. Team, Nucl. Fusion 47, L31 (2007)

[29] W. Daughton, J. Scudder, and H. Karimabadi, Phys. Plasmas 13, 2101 (2006).

[30] J. F. Drake, M. Swisdak, K. M. Schoeffler, B. N. Rogers, and S. Kobayashi, Geophys. Res. Lett. 33, 13105 (2006).

[31] H. Karimabadi, W. Daughton, and J. Scudder, Geophys. Res. Lett. 34, 13104 (2007).

[32] W. Daughton, V. Roytershteyn, B. J. Albright, H. Karimabadi, L. Yin, and K. J. Bowers, Phys. Rev. Lett. 103, 65004 (2009).

[33] W. Daughton, V. Roytershteyn, B. J. Albright, H. Karimabadi, L. Yin, and K. J. Bowers, Phys. Plasmas 16, 2117 (2009).

[34] L. S. Shepherd and P. A. Cassak, Phys. Rev. Lett. 105, 15004 (2010).

[35] Y. Huang, A. Bhattacharjee, and B. P. Sullivan, Phys. Plasmas 18, 2109 (2011).

[36] W. Park, D. A. Monticello, and R. B. White, Phys. Fluids 27, 137 (1984).

[37] R. S. Steinolfson and G. van Hoven, Phys. Fluids 27, 1207 (1984).

[38] L. C. Lee and Z. F. Fu, J. Geophys. Res. 91, 6807 (1986).

[39] D. Biskamp, Phys. Fluids 29, 1520 (1986)

[40] S. Jin and W. Ip, Phys. Fluids B 3, 1927 (1991)

[41] N. F. Loureiro, S. C. Cowley, W. D. Dorland, M. G. Haines, and A. A. Schekochihin, Phys. Rev. Lett. 95, 235003 (2005)

[42] G. Lapenta, Phys. Rev. Lett. 100, 235001 (2008)

[43] R. Samtaney, N. F. Loureiro, D. A. Uzdensky, A. A. Schekochihin, and S. C. Cowley, Phys. Rev. Lett. 103, 105004 (2009)

[44] N. F. Loureiro, D. A. Uzdensky, A. A. Schekochihin, S. C. Cowley, and T. A. Yousef, Mon. Not. R. Astron. Soc. 399, L146 (2009)

[45] A. Bhattacharjee, Y. Huang, H. Yang, and B. Rogers, Phys. Plasmas 16, 112102 (2009).

[46] P. A. Cassak, M. A. Shay, and J. F. Drake, Phys. Plasmas 16, 0702 (2009).

[47] Y. Huang and A. Bhattacharjee, Phys. Plasmas 17, 062104 (2010).

[48] M. Skender and G. Lapenta,
Phys. Plasmas 17, 2905 (2010)

[49] N. F. Loureiro, R. Samtaney, A. A. Schekochihin, and D. A. Uzdensky, Phys. Plasmas 19, 042303 (2012).

[50] S. S. Komissarov, M. Barkov, and M. Lyutikov, Mon. Not. R. Astron. Soc. 374, 415 (2007).

[51] S. Zenitani and M. Hoshino, Astrophys. J. 677, 530 (2008).

[52] W. Liu, H. Li, L. Yin, B. J. Albright, K. J. Bowers, and E. P. Liang, Phys. Plasmas 18, 052105 (2011)

[53] L. Sironi and A. Spitkovsky, Astrophys. J. 741, 39 (2011).

[54] B. Cerutti, G. R. Werner, D. A. Uzdensky, and M. C. Begelman, Astrophys. J. 754, L33 (2012).

[55] P. Riley, R. Lionello, Z. Miki, J. Linker, E. Clark, J. Lin, and Y. Ko, Astrophys. J. 655, 591 (2007).

[56] M. Bárta, B. Vršnak, and M. Karlický, Astron. Astrophys. 477, 649 (2008).

[57] L. Bettarini and G. Lapenta, Astron. Astrophys. 518, 57 (2010)

[58] M. Bárta, J. Büchner, M. Karlický, and J. Skála, Astrophys. J. 737, 24 (2011).

[59] H. Karimabadi, D. Krauss-Varban, N. Omidi, and H. X. Vu, J. Geophys. Res. 104, 12313 (1999)

[60] S. Jin, X. Hu, Q. Zong, S. Fu, B. Wilken, and J. Büchner, J. Geophys. Res. 106, 29807 (2001).

[61] A. P. Goodson, K. Böhm, and R. M. Winglee, Astrophys. J. 524, 142 (1999).

[62] A. P. Goodson and R. M. Winglee, Astrophys. J. 524, 159 (1999)

[63] D. A. Uzdensky, Astrophys. Space Sci. 292, 573 (2004)

[64] W. Fox, A. Bhattacharjee, and K. Germaschewski, Phys. Rev. Lett. 106, 215003 (2011).

[65] E. N. Parker, J. Geophys. Res. 62, PP. 509 (1957)

[66] P. A. Sweet, in Electromagnetic Phenomena in Cosmical Physics, IAU Symposium, Vol. 6, edited by B. Lehnert (1958) p. 123.

[67] H. E. Petschek, NASA Special Publication 50, 425 (1964).

[68] J. Birn, J. F. Drake, M. A. Shay, B. N. Rogers, R. E. Denton, M. Hesse, M. Kuznetsova, Z. W. Ma, A. Bhattacharjee, A. Otto, and P. L. Pritchett, J. Geophys. Res. 106, 3715 (2001).

[69] K. Shibata and S. Tanuma, Earth, Planets Space 53, 473 (2001)

[70] R. L. Fermo, J. F. Drake, and M. Swisdak, Phys. Plasmas 17, 010702 (2010)

[71] D. A. Uzdensky, N. F. Loureiro, and A. A. Schekochihin, Phys. Rev. Lett. 105, 235002 (2010)

[72] R. L. Fermo, J. F. Drake, M. Swisdak, and K. Hwang, J. Geophys. Res. 116, 09226 (2011)

[73] J. F. Drake, M. Swisdak, H. Che, and M. A. Shay, Nature 443, 553 (2006)

[74] M. Oka, M. Fujimoto, I. Shinohara, and T. D. Phan, J. Geophys. Res. 115, 08223 (2010)

[75] M. Oka, T. Phan, S. Krucker, M. Fujimoto, and I. Shinohara, Astrophys. J. 714, 915 (2010)

[76] N. F. Loureiro, A. A. Schekochihin, and S. C. Cowley, Phys. Plasmas 14, 0703 (2007)

[77] L. Ni, K. Germaschewski, Y. Huang, B. P. Sullivan, H. Yang, and A. Bhattacharjee, Phys. Plasmas 17, 052109 (2010)

[78] S. D. Baalrud, A. Bhattacharjee, and Y. Huang, Phys. Plasmas 19, 2101 (2012)

[79] S. D. Baalrud, A. Bhattacharjee, Y. Huang, and K. Germaschewski, Phys. Plasmas 18, 2108 (2011). 
[80] R. M. Kulsrud and S. W. Anderson, Astrophys. J. 396, 606 (1992)

[81] B. Coppi, R. Galvão, M. N. Rosenbluth, M. N., and P. Rutherford, Sov. J. Plasma Phys. 2, 533 (1976).

[82] A. A. Schekochihin, A. B. Iskakov, S. C. Cowley, J. C. McWilliams, M. R. E. Proctor, and T. A. Yousef, New J. Phys. 9, 300 (2007)

[83] A. Brandenburg and K. Subramanian, Phys. Rep. 417, 1 (2005)

[84] F. Porcelli, Phys. Fluids 30, 1734 (1987)

[85] S. Chandrasekhar, Hydrodynamic and Hydromagnetic Stabilit 9 . (Dover, Inc., 1981).

[86] S. I. Syrovatski, Sov. Phys. JETP 33, 933 (1971)

[87] H. R. Strauss, Phys. Fluids 19, 134 (1976).

[88] D. Biskamp, Nonlinear Magnetohydrodynamics (Cambridge University Press, 1993).

[89] One should note that, starting with the Syrovatskii solution [86], the literature is rich in ideal current sheet equilibrium solutions but, by definition, those cannot describe reconnection or the internal structure of the current sheet (see Ref. 2] for an extensive literature review on this subject). Families of solutions of the resistive equilibrium problem were derived by Craig and Henton [100], but none of these models the conventional SP-like configuration that we wish to treat here. Biskamp [88], employing the same power-series expansion as used here, avoids the closure problem by introducing an ad hoc guess for the functional form of the unreconnected magnetic field profile. In Appendix A we obtain an analytic equilibrium solution that is a $2 \mathrm{D}$ generalization of Biskamp's.

[90] The time dependence of $\kappa$ induced by the background shear flow becomes important at low values of $S$ and has been measured numerically in Ref. [77].

[91] Note that in the limit $\bar{y}_{0}=0$, we recover Eq. (15) of Paper I, with the exceptions that (i) here $f_{0}^{\prime}$ refers to a generic equilibrium and (ii) since the profiles are now continuous and we are no longer matching at a point, the subdominant contribution to $\Delta^{\prime}$ present in Paper I no longer appears in Eq. (78).

[92] D. A. Uzdensky and R. M. Kulsrud, Phys. Plasmas 5, 3249 (1998)

3] T. S. Hahm and R. M. Kulsrud, Phys. Fluids 28, 2412 (1985)

[94] A. Cole and R. Fitzpatrick, Phys. Plasmas 11, 3525 (2004)

[95] In theory, one could also look for solutions subject to the boundary condition $\Phi^{\prime}(0)=0$, which would yield $\Phi(\xi)=$ const. $\equiv \Phi_{0}$, and $\Psi(\xi)=f_{0}^{\prime} \xi \Phi_{0} / \lambda$. Such a solution, however, cannot be matched to the one obtained in the outer region, Eq. (60), unless $C_{2}^{ \pm}=0$, which implies $\Psi(\xi)=0$ in the entire domain.

[96] M. N. Rosenbluth, R. Y. Dagazian, and P. H. Rutherford, Phys. Fluids 16, 1894 (1973)

[97] R. L. Fermo, J. F. Drake, and M. Swisdak, Phys. Rev. Lett. 108, 255005 (2012).

[98] E. G. Harris, Nuovo Cimento 23, 115 (1962).

[99] These are the values of the coefficients obtained numerically in Ref. 92] for the smallest value of the magnetic Prandtl number employed in that paper, $P m=0.005$, and are assumed by us to be the converged values in the asymptotic case $P m \ll 1$.

[100] I. J. D. Craig and S. M. Henton, Astrophys. J. 450, 280 (1995) 